\title{
Systems of fractional Langevin equations of Riemann-Liouville and Hadamard types
}

\author{
Weerawat Sudsutad ${ }^{1,2}$, Sotiris K Ntouyas ${ }^{3,4}$ and Jessada Tariboon ${ }^{1,2^{*}}$
}

\author{
${ }^{*}$ Correspondence: \\ jessada.t@sci.kmutnb.ac.th \\ ${ }^{1}$ Nonlinear Dynamic Analysis \\ Research Center, Department of \\ Mathematics, Faculty of Applied \\ Science, King Mongkut's University \\ of Technology North Bangkok, \\ Bangkok, 10800, Thailand \\ ${ }^{2}$ Centre of Excellence in \\ Mathematics, CHE, Si Ayutthaya Rd. \\ Bangkok, 10400, Thailand \\ Full list of author information is \\ available at the end of the article
}

\begin{abstract}
Fractional differential equations have been shown to be very useful in the study of models of many phenomena in various fields of science and engineering, such as physics, chemistry, biology, signal and image processing, biophysics, blood flow phenomena, control theory, economics, aerodynamics, and fitting of experimental data. Much of the work on the topic deals with the governing equations involving Riemann-Liouville- and Caputo-type fractional derivatives. Another kind of fractional derivative is the Hadamard type, which was introduced in 1892. This derivative differs from the aforementioned derivatives in the sense that the kernel of the integral in the definition of the Hadamard derivative contains a logarithmic function of arbitrary exponent. In the present paper we introduce a new class of boundary value problems for Langevin fractional differential systems. The Langevin equation is widely used to describe the evolution of physical phenomena in fluctuating environments. We combine Riemann-Liouville- and Hadamard-type Langevin fractional differential equations subject to Hadamard and Riemann-Liouville fractional integral boundary conditions, respectively. Some new existence and uniqueness results for coupled and uncoupled systems are obtained by using fixed point theorems. The existence and uniqueness of solutions is established by Banach's contraction mapping principle, while the existence of solutions is derived by using the Leray-Schauder's alternative. The obtained results are well illustrated with the aid of examples.
\end{abstract}

MSC: $34 \mathrm{~A} 08 ; 34 \mathrm{~A} 12 ; 34 \mathrm{~B} 15$

Keywords: Riemann-Liouville fractional derivative; Hadamard fractional derivative; fractional integral boundary conditions; Langevin equation; coupled system; existence; uniqueness; fixed point theorems

\section{Introduction}

In this paper, we concentrate on the study of existence and uniqueness of solutions for a coupled systems of Riemann-Liouville and Hadamard fractional derivatives of Langevin equation with fractional integral conditions of the form

$$
\left\{\begin{array}{l}
{ }_{\mathrm{RL}} D^{q_{1}}\left({ }_{\mathrm{RL}} D^{p_{1}}+\lambda_{1}\right) x(t)=f(t, x(t), y(t)), \quad a \leq t \leq T, \\
{ }_{\mathrm{H}} D^{q_{2}}\left({ }_{\mathrm{H}} D^{p_{2}}+\lambda_{2}\right) y(t)=g(t, x(t), y(t)), \quad a \leq t \leq T, \\
x(a)=0, \quad \sigma_{1} x\left(\tau_{1}\right)=\sum_{i=1}^{m} \alpha_{i \mathrm{H}} I^{\rho_{i}} y\left(\eta_{i}\right), \\
y(a)=0, \quad \sigma_{2} y\left(\tau_{2}\right)=\sum_{j=1}^{n} \beta_{j \mathrm{RL}} I^{\gamma_{j}} x\left(\xi_{j}\right),
\end{array}\right.
$$

where ${ }_{\mathrm{RL}} D^{q},{ }_{\mathrm{H}} D^{p}$ are the Riemann-Liouville and Hadamard fractional derivative of orders $q$, $p$, respectively, when $q \in\left\{q_{1}, p_{1}\right\}$, and $p \in\left\{q_{2}, p_{2}\right\}$ with $0<q_{k}, p_{k}<1,1<q_{k}+p_{k}<2$,

(c) 2015 Sudsutad et al. This article is distributed under the terms of the Creative Commons Attribution 4.0 International License (http://creativecommons.org/licenses/by/4.0/), which permits unrestricted use, distribution, and reproduction in any medium, provided you give appropriate credit to the original author(s) and the source, provide a link to the Creative Commons license, and indicate if changes were made. 
$\lambda_{k}$ are given constants, $k=1,2,{ }_{\mathrm{RL}} I^{\gamma_{j}}{ }_{\mathrm{H}} I^{\rho_{i}}$ are the Riemann-Liouville and Hadamard fractional integral of orders $\gamma_{j}, \rho_{i}>0$, respectively, $\eta_{i}, \xi_{j} \in(a, T)$ and $\alpha_{i}, \beta_{j}, \sigma_{1}, \sigma_{2} \in \mathbb{R}$ for all $i=1,2, \ldots, m, j=1,2, \ldots, n, \tau_{1}, \tau_{2} \in(a, T], f, g:[a, T] \times \mathbb{R}^{2} \rightarrow \mathbb{R}$ are continuous functions.

Fractional differential equations have been shown to be very useful in the study of models of many phenomena in various fields of science and engineering, such as physics, chemistry, biology, signal and image processing, biophysics, blood flow phenomena, control theory, economics, aerodynamics, and fitting of experimental data. For examples and recent development of the topic, see [1-4] and references cited therein. Ahmad et al. [5-8] have studied the existence and uniqueness of solutions of nonlinear fractional differential and integro-differential equations for a variety of boundary conditions using standard fixed point theorems. Agarwal et al. [9] discusses the existence of solutions of fractional neutral functional differential equations. Baleanu et al. [10] considered $L^{p}$-solutions for a class of sequential fractional differential equations. In [11], the nonlinear alternative and Vitali convergence theorem were used for studying Caputo fractional boundary value problems with singularities in space variables. In Zhang et al. [12], the fixed point theory and monotone iterative technique were used to prove the existence of a unique solution for a class of nonlinear fractional integro-differential equations on semi-infinite domains in a Banach space. Liu et al. [13] discussed the existence of at least three solutions of $p$-Laplacian model involving the Caputo fractional derivative with Dirichlet-Neumann boundary conditions. However, it has been observed that most of the work on the topic involves either the Riemann-Liouville- or the Caputo-type fractional derivative.

Besides these derivatives, the Hadamard fractional derivative is another kind of fractional derivatives that was introduced by Hadamard in 1892 [14]. This fractional derivative differs from the other ones in the sense that the kernel of the integral (in the definition of Hadamard derivative) contains logarithmic function of arbitrary exponent. For background material of Hadamard fractional derivative and integral, we refer to [3, 15-19].

It seems that the abstract fractional differential equations involving Hadamard fractional derivatives and Hilfer-Hadamard fractional derivatives have not been fully explored so far. The basic information on various classes of abstract fractional equations and abstract Volterra integro-differential equations the interested reader can be found in [20-24] and the references cited therein.

The Langevin equation (first formulated by Langevin in 1908) is found to be an effective tool to describe the evolution of physical phenomena in fluctuating environments [25]. For some new developments on the fractional Langevin equation in physics; see, for example, [26-30]. Lizana et al. [31] have studied a single-particle equation of motion starting with a microscopic description of a tracer particle in a one-dimensional many-particle system with a general two-body interaction potential and they have shown that the resulting $d y-$ namical equation belongs to the class of fractional Langevin equations using a harmonization technique. In [32], Gambo et al. discussed the Caputo modification of the Hadamard fractional derivative. Ahmad et al. [33, 34] considered solutions of nonlinear Langevin equation involving two fractional orders. In [35], Tariboon et al. studied the existence and uniqueness of solutions of the nonlinear Langevin equation of Hadamard-Caputo-type fractional derivatives with nonlocal fractional integral conditions using a variety of fixed point theorems.

In this paper we prove the existence and uniqueness of the solutions by using Banach's contraction principle, and existence of solutions via Leray-Schauder's alternative. Exam- 
ples illustrating our results are also presented. The case of uncoupled systems is also discussed. We emphasize that in this paper we combine Riemann-Liouville- and Hadamardtype fractional differential equations subject to Hadamard and Riemann-Liouville fractional integral boundary conditions, respectively. To the best of the authors' knowledge this is the first paper dealing with systems with such combinations of equations and boundary conditions.

\section{Preliminaries}

In this section, we introduce some notations and definitions of fractional calculus and present preliminary results needed in our proofs later. To distinguish the different cases of derivatives and integrals we use the notations ${ }_{\mathrm{RL}} D,{ }_{\mathrm{RL}} I,{ }_{\mathrm{H}} D,{ }_{\mathrm{H}} I$ to denote RiemannLiouville or Hadamard derivative or integral respectively.

Definition 2.1 [3] The Riemann-Liouville fractional derivative of order $q>0$ of a continuous function $f:(a, \infty) \rightarrow \mathbb{R}, a>0$, is defined by

$$
{ }_{\mathrm{RL}} D^{q} f(t)=\frac{1}{\Gamma(n-q)}\left(\frac{d}{d t}\right)^{n} \int_{a}^{t}(t-s)^{n-q-1} f(s) d s, \quad n-1<q<n,
$$

where $n=[q]+1,[q]$ denotes the integer part of a real number $q$, provided the right-hand side is point-wise defined on $(a, \infty)$, where $\Gamma$ is the gamma function defined by $\Gamma(q)=$ $\int_{0}^{\infty} e^{-s} s^{q-1} d s$

Definition 2.2 [3] The Riemann-Liouville fractional integral of order $q>0$ of a continuous function $f:(a, \infty) \rightarrow \mathbb{R}, a>0$, is defined by

$$
\mathrm{RL}^{q} f(t)=\frac{1}{\Gamma(q)} \int_{a}^{t}(t-s)^{q-1} f(s) d s
$$

provided the right-hand side is point-wise defined on $(a, \infty)$.

Definition 2.3 [3] The Hadamard derivative of a measurable fractional order $q$ for a function $f:(a, \infty) \rightarrow \mathbb{R}, a>0$, is defined as

$$
{ }_{\mathrm{H}} D^{q} f(t)=\frac{1}{\Gamma(n-q)}\left(t \frac{d}{d t}\right)^{n} \int_{a}^{t}\left(\log \frac{t}{s}\right)^{n-q-1} \frac{f(s)}{s} d s, \quad n-1<q<n, n=[q]+1,
$$

where $\log (\cdot)=\log _{e}(\cdot)$, provided the (Lebesgue) integral exists and the operator $(t d / d t)^{n}$ can be applied.

Definition 2.4 [3] The Hadamard fractional integral of order $q \in \mathbb{R}^{+}$of a function $f(t)$, for all $0<a<t<\infty$, is defined as

$$
{ }_{\mathrm{H}} I^{q} f(t)=\frac{1}{\Gamma(q)} \int_{a}^{t}\left(\log \frac{t}{s}\right)^{q-1} f(s) \frac{d s}{s}
$$

provided the integral exists. 
Lemma 2.1 ([3], pp.71, 112, 114) Let $q>0, a>0$, and $\beta>0$. Then the following properties hold:

$$
\begin{aligned}
& \mathrm{RL}^{q}(t-a)^{\beta-1}(x)=\frac{\Gamma(\beta)}{\Gamma(\beta+q)}(x-a)^{\beta+q-1}, \\
& { }_{\mathrm{H}} I^{q}\left(\log \frac{t}{a}\right)^{\beta-1}(x)=\frac{\Gamma(\beta)}{\Gamma(\beta+q)}\left(\log \frac{x}{a}\right)^{\beta+q-1}, \\
& { }_{\mathrm{H}} I^{q} I^{\beta} f(x)={ }_{\mathrm{H}} I^{q+\beta} f(x) \quad \text { semigroup property. }
\end{aligned}
$$

Lemma 2.2 [36] Let $q>0$ and $x \in C(a, T) \cap L^{1}(a, T), a>0$. Then the fractional differential equation ${ }_{\mathrm{RL}} D^{q} x(t)=0$ has the solutions

$$
x(t)=\sum_{i=1}^{n} c_{i}(t-a)^{q-i}
$$

and the following formula holds:

$$
\mathrm{RL}^{q}{ }_{\mathrm{RL}} D^{q} x(t)=x(t)+\sum_{i=1}^{n} c_{i}(t-a)^{q-i},
$$

where $c_{i} \in \mathbb{R}, i=1,2, \ldots, n$, and $n-1<q<n$.

Lemma 2.3 [3, 36] Let $q>0$ and $x \in C(a, T) \cap L^{1}(a, T), a>0$. Then the Hadamard fractional differential equation ${ }_{\mathrm{H}} D^{q} x(t)=0$ has the solutions

$$
x(t)=\sum_{i=1}^{n} c_{i}\left(\log \frac{t}{a}\right)^{q-i},
$$

and the following formula holds:

$$
{ }_{\mathrm{H}}{ }^{q}{ }_{\mathrm{H}} D^{q} x(t)=x(t)+\sum_{i=1}^{n} c_{i}\left(\log \frac{t}{a}\right)^{q-i},
$$

where $c_{i} \in \mathbb{R}, i=1,2, \ldots, n$, and $n-1<q<n$.

In the following, for the sake of the convenience, we set

$$
\begin{array}{ll}
\Omega_{1}=\sum_{i=1}^{m} \frac{\alpha_{i}\left(\log \frac{\eta_{i}}{a}\right)^{q_{2}+p_{2}+\rho_{i}-1} \Gamma\left(q_{2}\right)}{\Gamma\left(q_{2}+p_{2}+\rho_{i}\right)}, & \Omega_{2}=\sum_{j=1}^{n} \frac{\beta_{j}\left(\xi_{j}-a\right)^{q_{1}+p_{1}+\gamma_{j}-1} \Gamma\left(q_{1}\right)}{\Gamma\left(q_{1}+p_{1}+\gamma_{j}\right)}, \\
\Omega_{3}=\frac{\sigma_{2} \Gamma\left(q_{2}\right)}{\Gamma\left(q_{2}+p_{2}\right)}\left(\log \frac{\tau_{2}}{a}\right)^{q_{2}+p_{2}-1}, & \Omega_{4}=\frac{\sigma_{1} \Gamma\left(q_{1}\right)}{\Gamma\left(q_{1}+p_{1}\right)}\left(\tau_{1}-a\right)^{q_{1}+p_{1}-1}
\end{array}
$$

and

$$
\Omega=\Omega_{1} \Omega_{2}-\Omega_{3} \Omega_{4}
$$

Lemma 2.4 Let $\Omega \neq 0,0<q_{k}, p_{k}<1,1<q_{k}+p_{k}<2, k=1,2, \rho_{i}, \gamma_{j}>0, \alpha_{i}, \beta_{j}, \sigma_{1}, \sigma_{2} \in \mathbb{R}$, $\eta_{i}, \xi_{j} \in(a, T), i=1,2, \ldots, m, j=1,2, \ldots, n, \tau_{1}, \tau_{2} \in(a, T]$, and $\phi, \psi \in C([a, T], \mathbb{R}), a>0$. Then 
the problem

$$
\left\{\begin{array}{l}
\mathrm{RL} D^{q_{1}}\left(\mathrm{RL}_{\mathrm{L}} D^{p_{1}}+\lambda_{1}\right) x(t)=\phi(t), \quad a \leq t \leq T, \\
{ }_{\mathrm{H}} D^{q_{2}}\left({ }_{\mathrm{H}} D^{p_{2}}+\lambda_{2}\right) y(t)=\psi(t), \quad a \leq t \leq T, \\
x(a)=0, \quad \sigma_{1} x\left(\tau_{1}\right)=\sum_{i=1}^{m} \alpha_{i \mathrm{H}} I^{\rho_{i}} y\left(\eta_{i}\right), \\
y(a)=0, \quad \sigma_{2} y\left(\tau_{2}\right)=\sum_{j=1}^{n} \beta_{j \mathrm{RL}} I^{\gamma_{j}} x\left(\xi_{j}\right),
\end{array}\right.
$$

has a solution if and only if the system

$$
\begin{aligned}
x(t)= & { }_{\mathrm{RL}} I^{q_{1}+p_{1}} \phi(t)-\lambda_{1 \mathrm{RL}} I^{p_{1}} x(t)-\frac{(t-a)^{q_{1}+p_{1}-1} \Gamma\left(q_{1}\right)}{\Omega \Gamma\left(q_{1}+p_{1}\right)}\left[\left(\sum_{j=1}^{n} \beta_{j \mathrm{RL}} I^{q_{1}+p_{1}+\gamma_{j}} \phi\left(\xi_{j}\right)\right.\right. \\
& \left.-\lambda_{1} \sum_{j=1}^{n} \beta_{j \mathrm{RL}} I^{p_{1}+\gamma_{j}} x\left(\xi_{j}\right)+\lambda_{2} \sigma_{2 \mathrm{H}} I^{p_{2}} y\left(\tau_{2}\right)-\sigma_{2 \mathrm{H}} I^{q_{2}+p_{2}} \psi\left(\tau_{2}\right)\right) \Omega_{1} \\
& +\left(\sum_{i=1}^{m} \alpha_{i \mathrm{H}} I^{q_{2}+p_{2}+\rho_{i}} \psi\left(\eta_{i}\right)-\lambda_{2} \sum_{i=1}^{m} \alpha_{i \mathrm{H}} I^{p_{2}+\rho_{i}} y\left(\eta_{i}\right)+\lambda_{1} \sigma_{1 \mathrm{RL}} I^{p_{1}} x\left(\tau_{1}\right)\right. \\
& \left.\left.-\sigma_{1 \mathrm{RL}} I^{q_{1}+p_{1}} \phi\left(\tau_{1}\right)\right) \Omega_{3}\right]
\end{aligned}
$$

and

$$
\begin{aligned}
y(t)= & { }_{\mathrm{H}} I^{q_{2}+p_{2}} \psi(t)-\lambda_{2 \mathrm{H}} I^{p_{2}} y(t)-\frac{\left(\log \frac{t}{a}\right)^{q_{2}+p_{2}-1} \Gamma\left(q_{2}\right)}{\Omega \Gamma\left(q_{2}+p_{2}\right)}\left[\left(\sum_{i=1}^{m} \alpha_{i \mathrm{H}} I^{q_{2}+p_{2}+\rho_{i}} \psi\left(\eta_{i}\right)\right.\right. \\
& \left.-\lambda_{2} \sum_{i=1}^{m} \alpha_{i \mathrm{H}} I^{p_{2}+\rho_{i}} y\left(\eta_{i}\right)+\lambda_{1} \sigma_{1 \mathrm{RL}} I^{p_{1}} x\left(\tau_{1}\right)-\sigma_{1 \mathrm{RL}} I^{q_{1}+p_{1}} \phi\left(\tau_{1}\right)\right) \Omega_{2} \\
& +\left(\sum_{j=1}^{n} \beta_{j \mathrm{RL}} I^{q_{1}+p_{1}+\gamma_{j}} \phi\left(\xi_{j}\right)-\lambda_{1} \sum_{j=1}^{n} \beta_{j \mathrm{RL}} I^{p_{1}+\gamma_{j}} x\left(\xi_{j}\right)+\lambda_{2} \sigma_{2 \mathrm{H}} I^{p_{2}} y\left(\tau_{2}\right)\right. \\
& \left.\left.-\sigma_{2 \mathrm{H}} I^{q_{2}+p_{2}} \psi\left(\tau_{2}\right)\right) \Omega_{4}\right]
\end{aligned}
$$

has a solution.

Proof Using Lemmas 2.2 and 2.3 and the first two equations in (1.1) can be expressed as equivalent integral equations

$$
\begin{aligned}
& \left(\mathrm{RL} D^{p_{1}}+\lambda_{1}\right) x(t)={ }_{\mathrm{RL}} I^{q_{1}} \phi(t)-c_{1}(t-a)^{q_{1}-1}, \\
& \left({ }_{\mathrm{H}} D^{p_{2}}+\lambda_{2}\right) y(t)={ }_{\mathrm{H}} I^{q_{2}} \psi(t)-d_{1}\left(\log \frac{t}{a}\right)^{q_{2}-1} .
\end{aligned}
$$

It follows that

$$
x(t)={ }_{\mathrm{RL}} I^{q_{1}+p_{1}} \phi(t)-\lambda_{1 \mathrm{RL}} I^{p_{1}} x(t)-c_{1} \frac{\Gamma\left(q_{1}\right)(t-a)^{q_{1}+p_{1}-1}}{\Gamma\left(q_{1}+p_{1}\right)}-c_{2}(t-a)^{p_{1}-1}
$$


and

$$
y(t)={ }_{\mathrm{H}} I^{q_{2}+p_{2}} \psi(t)-\lambda_{2 \mathrm{H}} I^{p_{2}} y(t)-d_{1} \frac{\Gamma\left(q_{2}\right)\left(\log \frac{t}{a}\right)^{q_{2}+p_{2}-1}}{\Gamma\left(q_{2}+p_{2}\right)}-d_{2}\left(\log \frac{t}{a}\right)^{p_{2}-1}
$$

where $c_{1}, c_{2}, d_{1}, d_{2} \in \mathbb{R}$. The condition $x(a)=y(a)=0$ implies that $c_{2}=d_{2}=0$.

Taking the Riemann-Liouville and Hadamard fractional integrals of order $\gamma_{j}, \rho_{i}>0$ for (2.4) and (2.5), respectively, and using the property given in Lemma 2.1, we obtain

$$
\begin{aligned}
& \sigma_{2 \mathrm{H}} I^{q_{2}+p_{2}} \psi\left(\tau_{2}\right)-\lambda_{2} \sigma_{2 \mathrm{H}} I^{p_{2}} y\left(\tau_{2}\right)-d_{1} \Omega_{3} \\
& =\sum_{j=1}^{n} \beta_{j \mathrm{RL}} I^{q_{1}+p_{1}+\gamma_{j}} \phi\left(\xi_{j}\right)-\lambda_{1} \sum_{j=1}^{n} \beta_{j \mathrm{RL}} I^{p_{1}+\gamma_{j}} x\left(\xi_{j}\right)-c_{1} \Omega_{2}, \\
& \sigma_{1 \mathrm{RL}} I^{q_{1}+p_{1}} \phi\left(\tau_{1}\right)-\lambda_{1} \sigma_{1 \mathrm{RL}} I^{p_{1}} x\left(\tau_{1}\right)-c_{1} \Omega_{4} \\
& =\sum_{i=1}^{m} \alpha_{i \mathrm{H}} I^{q_{2}+p_{2}+\rho_{i}} \psi\left(\eta_{i}\right)-\lambda_{2} \sum_{i=1}^{m} \alpha_{i \mathrm{H}} I^{p_{2}+\rho_{i}} y\left(\eta_{i}\right)-d_{1} \Omega_{1} .
\end{aligned}
$$

Solving the above system of linear equations for constants $c_{1}$ and $d_{1}$, we get

$$
\begin{aligned}
c_{1}= & \frac{\Omega_{1}}{\Omega}\left(\sum_{j=1}^{n} \beta_{j \mathrm{RL}} I^{q_{1}+p_{1}+\gamma_{j}} \phi\left(\xi_{j}\right)-\lambda_{1} \sum_{j=1}^{n} \beta_{j \mathrm{RL}} I^{p_{1}+\gamma_{j}} x\left(\xi_{j}\right)+\lambda_{2} \sigma_{2 \mathrm{H}} I^{p_{2}} y\left(\tau_{2}\right)\right. \\
& \left.-\sigma_{2 \mathrm{H}} I^{q_{2}+p_{2}} \psi\left(\tau_{2}\right)\right)+\frac{\Omega_{3}}{\Omega}\left(\sum_{i=1}^{m} \alpha_{i \mathrm{H}} I^{q_{2}+p_{2}+\rho_{i}} \psi\left(\eta_{i}\right)-\lambda_{2} \sum_{i=1}^{m} \alpha_{i \mathrm{H}} I^{p_{2}+\rho_{i}} y\left(\eta_{i}\right)\right. \\
& \left.+\lambda_{1} \sigma_{1 \mathrm{RL}} I^{p_{1}} x\left(\tau_{1}\right)-\sigma_{1 \mathrm{RL}} I^{q_{1}+p_{1}} \phi\left(\tau_{1}\right)\right) \\
d_{1}= & \frac{\Omega_{2}}{\Omega}\left(\sum_{i=1}^{m} \alpha_{i \mathrm{H}} I^{q_{2}+p_{2}+\rho_{i}} \psi\left(\eta_{i}\right)-\lambda_{2} \sum_{i=1}^{m} \alpha_{i \mathrm{H}} I^{p_{2}+\rho_{i}} y\left(\eta_{i}\right)+\lambda_{1} \sigma_{1 \mathrm{RL}} I^{p_{1}} x\left(\tau_{1}\right)\right. \\
& \left.-\sigma_{1 \mathrm{RL}} I^{q_{1}+p_{1}} \phi\left(\tau_{1}\right)\right)+\frac{\Omega_{4}}{\Omega}\left(\sum_{j=1}^{n} \beta_{j \mathrm{RL}} I^{q_{1}+p_{1}+\gamma_{j}} \phi\left(\xi_{j}\right)-\lambda_{1} \sum_{j=1}^{n} \beta_{j \mathrm{RL}} I^{p_{1}+\gamma_{j}} x\left(\xi_{j}\right)\right. \\
& \left.+\lambda_{2} \sigma_{2 \mathrm{H}} I^{p_{2}} y\left(\tau_{2}\right)-\sigma_{2 \mathrm{H}} I^{q_{2}+p_{2}} \psi\left(\tau_{2}\right)\right) .
\end{aligned}
$$

Substituting the values of $c_{1}, c_{2}, d_{1}$, and $d_{2}$ in (2.4) and (2.5), we obtain the expressions (2.2) and (2.3).

\section{Main results}

Throughout this paper, for convenience, we use the following expressions:

$$
\mathrm{RL} I^{w} h(s, x(s), y(s))(v)=\frac{1}{\Gamma(w)} \int_{a}^{v}(v-s)^{w-1} h(s, x(s), y(s)) d s
$$

and

$$
{ }_{\mathrm{H}} I^{u} h(s, x(s), y(s))(v)=\frac{1}{\Gamma(u)} \int_{a}^{v}\left(\log \frac{v}{s}\right)^{u-1} \frac{h(s, x(s), y(s))}{s} d s,
$$


where $u \in\left\{q_{2}, p_{2}, \gamma_{j}\right\}, w \in\left\{q_{1}, p_{1}, \rho_{i}\right\}, v \in\left\{t, \tau_{1}, \tau_{2}, \eta_{i}, \xi_{j}\right\}$ and $h=\{f, g\}, i=1,2, \ldots, m, j=$ $1,2, \ldots, n$.

Let $\mathcal{C}=C([a, T], \mathbb{R})$ denote the Banach space of all continuous functions from $[a, T]$ to $\mathbb{R}$. Let us introduce the space $X=\{x(t) \mid x(t) \in C([a, T])\}$ endowed with the norm $\|x\|=$ $\sup \{|x(t)|, t \in[a, T]\}$. Obviously $(X,\|\cdot\|)$ is a Banach space. In addition the product space $(X \times X,\|(x, y)\|)$ is a Banach space with norm $\|(x, y)\|=\|x\|+\|y\|$.

Definition 3.1 A $(x, y) \in X \times X$ is said to be a solution of the system (1.1) if $(x, y)$ satisfies the system $_{\mathrm{RL}} D^{q_{1}}\left({ }_{\mathrm{RL}} D^{p_{1}}+\lambda_{1}\right) x(t)=f(t, x(t), y(t)),{ }_{\mathrm{H}} D^{q_{2}}\left({ }_{\mathrm{H}} D^{p_{2}}+\lambda_{2}\right) y(t)=g(t, x(t), y(t))$, on $[a, T]$, and the conditions $x(a)=0, \sigma_{1} x\left(\tau_{1}\right)=\sum_{i=1}^{m} \alpha_{i \mathrm{H}} I^{\rho_{i}} y\left(\eta_{i}\right), y(a)=0, \sigma_{2} y\left(\tau_{2}\right)=\sum_{j=1}^{n} \beta_{j \mathrm{RL}} I^{\gamma_{j}} x\left(\xi_{j}\right)$.

In view of Lemma 2.4, we define an operator $\mathcal{Q}: X \times X \rightarrow X \times X$ by

$$
\mathcal{Q}(x, y)(t)=\left(\begin{array}{l}
\mathcal{Q}_{1}(x, y)(t) \\
\mathcal{Q}_{2}(x, y)(t)
\end{array}\right),
$$

where

$$
\begin{aligned}
& \mathcal{Q}_{1}(x, y)(t) \\
&=\mathrm{RL}^{q_{1}+p_{1}} f(s, x(s), y(s))(t)-\lambda_{1 \mathrm{RL}} I^{p_{1}} x(t) \\
& \quad-\frac{(t-a)^{q_{1}+p_{1}-1} \Gamma\left(q_{1}\right)}{\Omega \Gamma\left(q_{1}+p_{1}\right)}\left[\left(\sum_{j=1}^{n} \beta_{j \mathrm{RL}} I^{q_{1}+p_{1}+\gamma_{j}} f(s, x(s), y(s))\left(\xi_{j}\right)\right.\right. \\
&\left.\quad-\lambda_{1} \sum_{j=1}^{n} \beta_{j \mathrm{RL}} I^{p_{1}+\gamma_{j}} x\left(\xi_{j}\right)+\lambda_{2} \sigma_{2 \mathrm{H}} I^{p_{2}} y\left(\tau_{2}\right)-\sigma_{2 \mathrm{H}} I^{q_{2}+p_{2}} g(s, x(s), y(s))\left(\tau_{2}\right)\right) \Omega_{1} \\
& \quad+\left(\sum_{i=1}^{m} \alpha_{i \mathrm{H}} I^{q_{2}+p_{2}+\rho_{i}} g(s, x(s), y(s))\left(\eta_{i}\right)-\lambda_{2} \sum_{i=1}^{m} \alpha_{i \mathrm{H}} I^{p_{2}+\rho_{i}} y\left(\eta_{i}\right)+\lambda_{1} \sigma_{1 \mathrm{RL}} I^{p_{1}} x\left(\tau_{1}\right)\right. \\
&\left.\left.\quad-\sigma_{1 \mathrm{RL}} I^{q_{1}+p_{1}} f(s, x(s), y(s))\left(\tau_{1}\right)\right) \Omega_{3}\right]
\end{aligned}
$$

and

$$
\begin{aligned}
\mathcal{Q}_{2}(x, y)(t) & { }_{\mathrm{H}} I^{q_{2}+p_{2}} g(s, x(s), y(s))(t)-\lambda_{2 \mathrm{H}} I^{p_{2}} y(t) \\
& -\frac{\left(\log \frac{t}{a}\right)^{q_{2}+p_{2}-1} \Gamma\left(q_{2}\right)}{\Omega \Gamma\left(q_{2}+p_{2}\right)}\left[\left(\sum_{i=1}^{m} \alpha_{i \mathrm{H}} I^{q_{2}+p_{2}+\rho_{i}} g(s, x(s), y(s))\left(\eta_{i}\right)\right.\right. \\
& \left.-\lambda_{2} \sum_{i=1}^{m} \alpha_{i \mathrm{H}} I^{p_{2}+\rho_{i}} y\left(\eta_{i}\right)+\lambda_{1} \sigma_{1 \mathrm{RL}} I^{p_{1}} x\left(\tau_{1}\right)-\sigma_{1 \mathrm{RL}} I^{q_{1}+p_{1}} f(s, x(s), y(s))\left(\tau_{1}\right)\right) \Omega_{2} \\
& +\left(\sum_{j=1}^{n} \beta_{j \mathrm{RL}} I^{q_{1}+p_{1}+\gamma_{j}} f(s, x(s), y(s))\left(\xi_{j}\right)-\lambda_{1} \sum_{j=1}^{n} \beta_{j \mathrm{RL}} I^{p_{1}+\gamma_{j}} x\left(\xi_{j}\right)\right. \\
& \left.\left.+\lambda_{2} \sigma_{2 \mathrm{H}} I^{p_{2}} y\left(\tau_{2}\right)-\sigma_{2 \mathrm{H}} I^{q_{2}+p_{2}} g(s, x(s), y(s))\left(\tau_{2}\right)\right) \Omega_{4}\right] .
\end{aligned}
$$


For the sake of convenience, we set

$$
\begin{aligned}
& A_{1}=\frac{(T-a)^{q_{1}+p_{1}-1} \Gamma\left(q_{1}\right)}{\Gamma\left(q_{1}+p_{1}\right)}, \quad A_{2}=\frac{\left(\log \frac{T}{a}\right)^{q_{2}+p_{2}-1} \Gamma\left(q_{2}\right)}{\Gamma\left(q_{2}+p_{2}\right)} \\
& A_{3}=\frac{(T-a)^{p_{1}}}{\Gamma\left(p_{1}+1\right)}, \quad A_{4}=\frac{(T-a)^{q_{1}+p_{1}}}{\Gamma\left(q_{1}+p_{1}+1\right)}, \\
& A_{5}=\frac{\left(\tau_{1}-a\right)^{p_{1}}}{\Gamma\left(p_{1}+1\right)}, \quad A_{6}=\frac{\left(\tau_{1}-a\right)^{q_{1}+p_{1}}}{\Gamma\left(q_{1}+p_{1}+1\right)}, \\
& A_{7}=\frac{\left(\log \frac{T}{a}\right)^{p_{2}}}{\Gamma\left(p_{2}+1\right)}, \quad A_{8}=\frac{\left(\log \frac{T}{a}\right)^{q_{2}+p_{2}}}{\Gamma\left(q_{2}+p_{2}+1\right)}, \\
& A_{9}=\frac{\left(\log \frac{\tau_{2}}{a}\right)^{p_{2}}}{\Gamma\left(p_{2}+1\right)}, \quad A_{10}=\frac{\left(\log \frac{\tau_{2}}{a}\right)^{q_{2}+p_{2}}}{\Gamma\left(q_{2}+p_{2}+1\right)}, \\
& A_{11}=\sum_{i=1}^{m} \frac{\left|\alpha_{i}\right|\left(\log \frac{\eta_{i}}{a}\right)^{p_{2}+\rho_{i}}}{\Gamma\left(p_{2}+\rho_{i}+1\right)}, \quad A_{12}=\sum_{i=1}^{m} \frac{\left|\alpha_{i}\right|\left(\log \frac{\eta_{i}}{a}\right)^{q_{2}+p_{2}+\rho_{i}}}{\Gamma\left(q_{2}+p_{2}+\rho_{i}+1\right)} \\
& A_{13}=\sum_{j=1}^{n} \frac{\left|\beta_{j}\right|\left(\xi_{j}-a\right)^{p_{1}+\gamma_{j}}}{\Gamma\left(p_{1}+\gamma_{j}+1\right)}, \quad A_{14}=\sum_{j=1}^{n} \frac{\left|\beta_{j}\right|\left(\xi_{j}-a\right)^{q_{1}+p_{1}+\gamma_{j}}}{\Gamma\left(q_{1}+p_{1}+\gamma_{j}+1\right)}
\end{aligned}
$$

The first result is concerned with the existence and uniqueness of solutions for the problem (1.1) and is based on Banach's fixed point theorem.

Theorem 3.1 Assume that $f, g:[a, T] \times \mathbb{R}^{2} \rightarrow \mathbb{R}$ are continuous functions and there exist constants $m_{i}, n_{i}, i=1,2$ such that for all $t \in[a, T], a>0$, and $x_{i}, y_{i} \in \mathbb{R}, i=1,2$,

$$
\left|f\left(t, x_{1}, y_{1}\right)-f\left(t, x_{2}, y_{2}\right)\right| \leq m_{1}\left|x_{1}-x_{2}\right|+m_{2}\left|y_{1}-y_{2}\right|
$$

and

$$
\left|g\left(t, x_{1}, y_{1}\right)-g\left(t, x_{2}, y_{2}\right)\right| \leq n_{1}\left|x_{1}-x_{2}\right|+n_{2}\left|y_{1}-y_{2}\right|
$$

In addition, assume that

$$
B_{1}+C_{1}<1
$$

where

$$
\begin{aligned}
& M_{1}=\frac{A_{1}}{|\Omega|}\left(\left|\sigma_{1}\right|\left|\Omega_{3}\right| A_{6}+\left|\Omega_{1}\right| A_{14}+A_{4}\right), \\
& M_{2}=\frac{A_{1}}{|\Omega|}\left(\left|\sigma_{2}\right|\left|\Omega_{1}\right| A_{10}+\left|\Omega_{3}\right| A_{12}\right), \\
& M_{3}=\frac{\left|\lambda_{1}\right| A_{1}}{|\Omega|}\left(\left|\sigma_{1}\right|\left|\Omega_{3}\right| A_{5}+\left|\Omega_{1}\right| A_{13}\right)+\left|\lambda_{1}\right| A_{3}, \\
& M_{4}=\frac{\left|\lambda_{2}\right| A_{1}}{|\Omega|}\left(\left|\sigma_{2}\right|\left|\Omega_{1}\right| A_{9}+\left|\Omega_{3}\right| A_{11}\right), \\
& M_{5}=\frac{A_{2}}{|\Omega|}\left(\left|\sigma_{2}\right|\left|\Omega_{4}\right| A_{10}+\left|\Omega_{2}\right| A_{12}+A_{8}\right),
\end{aligned}
$$




$$
\begin{aligned}
& M_{6}=\frac{A_{2}}{|\Omega|}\left(\left|\sigma_{1}\right|\left|\Omega_{2}\right| A_{6}+\left|\Omega_{4}\right| A_{14}\right), \\
& M_{7}=\frac{\left|\lambda_{2}\right| A_{2}}{|\Omega|}\left(\left|\sigma_{2}\right|\left|\Omega_{4}\right| A_{9}+\left|\Omega_{2}\right| A_{11}\right)+\left|\lambda_{2}\right| A_{7}, \\
& M_{8}=\frac{\left|\lambda_{1}\right| A_{2}}{|\Omega|}\left(\left|\sigma_{1}\right|\left|\Omega_{2}\right| A_{5}+\left|\Omega_{4}\right| A_{13}\right)
\end{aligned}
$$

and

$$
\begin{aligned}
& B_{1}=\left(m_{1}+m_{2}\right) M_{1}+\left(n_{1}+n_{2}\right) M_{2}+M_{3}+M_{4}, \\
& C_{1}=\left(m_{1}+m_{2}\right) M_{6}+\left(n_{1}+n_{2}\right) M_{5}+M_{7}+M_{8} .
\end{aligned}
$$

Then the boundary value problem (1.1) has a unique solution on $[a, T]$.

Proof Define $\sup _{t \in[a, T]} f(t, 0,0)=N_{1}<\infty$ and $\sup _{t \in[a, T]} g(t, 0,0)=N_{2}<\infty$ and choose a positive real number $r$, such that

$$
r \geq \max \left\{\frac{M_{1} N_{1}+M_{2} N_{2}}{1-B_{1}}, \frac{M_{6} N_{1}+M_{5} N_{2}}{1-C_{1}}\right\}
$$

First, we show that $\mathcal{Q} B_{r} \subset B_{r}$, where $B_{r}=\{(x, y) \in X \times X:\|(x, y)\| \leq r\}$. For $(x, y) \in B_{r}$, we have

$$
\begin{aligned}
& \left|\mathcal{Q}_{1}(x, y)(t)\right| \\
& \leq \sup _{t \in[a, T]}\left\{{ }_{R L} I^{q_{1}+p_{1}}|f(s, x(s), y(s))|(t)+\left|\lambda_{1}\right|_{\mathrm{RL}} I^{p_{1}}|x(s)|(t)+\frac{(t-a)^{q_{1}+p_{1}-1} \Gamma\left(q_{1}\right)}{|\Omega| \Gamma\left(q_{1}+p_{1}\right)}\right. \\
& \quad \times\left(\left(\sum_{j=1}^{n}\left|\beta_{j}\right|_{\mathrm{RL}} I^{q_{1}+p_{1}+\gamma_{j}}|f(s, x(s), y(s))|\left(\xi_{j}\right)+\left|\lambda_{1}\right| \sum_{j=1}^{n}\left|\beta_{j}\right|_{\mathrm{RL}} I^{p_{1}+\gamma_{j}}|x(s)|\left(\xi_{j}\right)\right.\right. \\
& \left.\quad+\left|\lambda_{2}\right|\left|\sigma_{2}\right|_{\mathrm{H}} I^{p_{2}}|y(s)|\left(\tau_{2}\right)+\left|\sigma_{2}\right|_{\mathrm{H}} I^{q_{2}+p_{2}}|g(s, x(s), y(s))|\left(\tau_{2}\right)\right)\left|\Omega_{1}\right| \\
& \quad+\left(\sum_{i=1}^{m}\left|\alpha_{i}\right|_{\mathrm{H}} I^{q_{2}+p_{2}+\rho_{i}}|g(s, x(s), y(s))|\left(\eta_{i}\right)+\left|\lambda_{2}\right| \sum_{i=1}^{m}\left|\alpha_{i}\right|_{\mathrm{H}} I^{p_{2}+\rho_{i}}|y(s)|\left(\eta_{i}\right)\right. \\
& \left.\left.\left.\quad+\left|\lambda_{1}\right|\left|\sigma_{1}\right|_{\mathrm{RL}} I^{p_{1}}|x(s)|\left(\tau_{1}\right)+\left|\sigma_{1}\right|_{\mathrm{RL}} I^{q_{1}+p_{1}}|f(s, x(s), y(s))|\left(\tau_{1}\right)\right)\left|\Omega_{3}\right|\right)\right\} \\
& \leq \mathrm{RL}^{q_{1}+p_{1}}(|f(s, x(s), y(s))-f(s, 0,0)|+|f(s, 0,0)|)(T)+\left|\lambda_{1}\right|_{\mathrm{RL}} I^{p_{1}}|x(s)|(T) \\
& \quad+\frac{(T-a)^{q_{1}+p_{1}-1} \Gamma\left(q_{1}\right)}{|\Omega| \Gamma\left(q_{1}+p_{1}\right)}\left(\left(\sum_{j=1}^{n}\left|\beta_{j}\right|_{\mathrm{RL}} I^{q_{1}+p_{1}+\gamma_{j}}(|f(s, x(s), y(s))-f(s, 0,0)|\right.\right. \\
& \quad+|f(s, 0,0)|)\left(\xi_{j}\right)+\left|\lambda_{1}\right| \sum_{j=1}^{n}\left|\beta_{j}\right|_{\mathrm{RL}} I^{p_{1}+\gamma_{j}}|x(s)|\left(\xi_{j}\right)+\left|\lambda_{2}\right|\left|\sigma_{2}\right|_{\mathrm{H}} I^{p_{2}}|y(s)|\left(\tau_{2}\right) \\
& \left.+\left|\sigma_{2}\right| I^{q_{2}+p_{2}}(|g(s, x(s), y(s))-g(s, 0,0)|+|g(s, 0,0)|)\left(\tau_{2}\right)\right)\left|\Omega_{1}\right|
\end{aligned}
$$




$$
\begin{aligned}
& +\left(\sum_{i=1}^{m}\left|\alpha_{i}\right|_{\mathrm{H}} I^{q_{2}+p_{2}+\rho_{i}}(|g(s, x(s), y(s))-g(s, 0,0)|+|g(s, 0,0)|)\left(\eta_{i}\right)\right. \\
& +\left|\lambda_{2}\right| \sum_{i=1}^{m}\left|\alpha_{i}\right|_{\mathrm{H}} I^{p_{2}+\rho_{i}}|y(s)|\left(\eta_{i}\right)+\left|\lambda_{1}\right|\left|\sigma_{1}\right|_{\mathrm{RL}} I^{p_{1}}|x(s)|\left(\tau_{1}\right) \\
& \left.\left.+\left|\sigma_{1}\right|_{\mathrm{RL}} I^{q_{1}+p_{1}}(|f(s, x(s), y(s))-f(s, 0,0)|+|f(s, 0,0)|)\left(\tau_{1}\right)\right)\left|\Omega_{3}\right|\right) \\
& \leq\left(m_{1}\|x\|+m_{2}\|y\|+N_{1}\right)_{\mathrm{RL}} I^{q_{1}+p_{1}}(1)(T)+\left|\lambda_{1}\right|\|x\|_{\mathrm{RL}} I^{p_{1}}(1)(T) \\
& +\frac{(T-a)^{q_{1}+p_{1}-1} \Gamma\left(q_{1}\right)}{|\Omega| \Gamma\left(q_{1}+p_{1}\right)}\left(\left(\left(m_{1}\|x\|+m_{2}\|y\|+N_{1}\right) \sum_{j=1}^{n}\left|\beta_{j}\right|_{\mathrm{RL}} I^{q_{1}+p_{1}+\gamma_{j}}(1)\left(\xi_{j}\right)\right.\right. \\
& +\left|\lambda_{1}\right|\|x\| \sum_{j=1}^{n}\left|\beta_{j}\right|_{\mathrm{RL}} I^{p_{1}+\gamma_{j}}(1)\left(\xi_{j}\right)+\left|\lambda_{2}\right|\left|\sigma_{2}\right|\|y\|_{\mathrm{H}} I^{p_{2}}(1)\left(\tau_{2}\right) \\
& \left.+\left|\sigma_{2}\right|\left(n_{1}\|x\|+n_{2}\|y\|+N_{2}\right)_{\mathrm{H}} I^{q_{2}+p_{2}}(1)\left(\tau_{2}\right)\right)\left|\Omega_{1}\right| \\
& +\left(\left(n_{1}\|x\|+n_{2}\|y\|+N_{2}\right) \sum_{i=1}^{m}\left|\alpha_{i}\right|_{\mathrm{H}} I^{q_{2}+p_{2}+\rho_{i}}(1)\left(\eta_{i}\right)+\left|\lambda_{2}\right|\|y\| \sum_{i=1}^{m}\left|\alpha_{i}\right|_{\mathrm{H}} I^{p_{2}+\rho_{i}}(1)\left(\eta_{i}\right)\right. \\
& \left.\left.+\left|\lambda_{1}\right|\left|\sigma_{1}\right|\|x\|_{\mathrm{RL}} I^{p_{1}}(1)\left(\tau_{1}\right)+\left|\sigma_{1}\right|\left(m_{1}\|x\|+m_{2}\|y\|+N_{1}\right)_{\mathrm{RL}} I^{q_{1}+p_{1}}(1)\left(\tau_{1}\right)\right)\left|\Omega_{3}\right|\right) \\
& =\left(\frac{(T-a)^{q_{1}+p_{1}-1} \Gamma\left(q_{1}\right)}{|\Omega| \Gamma\left(q_{1}+p_{1}\right)}\left(\frac{\left|\Omega_{3}\right|\left|\sigma_{1}\right|\left(\tau_{1}-a\right)^{q_{1}+p_{1}}}{\Gamma\left(q_{1}+p_{1}+1\right)}+\sum_{j=1}^{n} \frac{\left|\Omega_{1}\right|\left|\beta_{j}\right|\left(\xi_{j}-a\right)^{q_{1}+p_{1}+\gamma_{j}}}{\Gamma\left(q_{1}+p_{1}+\gamma_{j}+1\right)}\right)\right. \\
& \left.+\frac{(T-a)^{q_{1}+p_{1}}}{\Gamma\left(q_{1}+p_{1}+1\right)}\right)\left(m_{1}\|x\|+m_{2}\|y\|+N_{1}\right)+\frac{(T-a)^{q_{1}+p_{1}-1} \Gamma\left(q_{1}\right)}{|\Omega| \Gamma\left(q_{1}+p_{1}\right)} \\
& \times\left(\frac{\left|\sigma_{2}\right|\left|\Omega_{1}\right|\left(\log \frac{\tau_{2}}{a}\right)^{q_{2}+p_{2}}}{\Gamma\left(q_{2}+p_{2}+1\right)}+\sum_{i=1}^{m} \frac{\left|\Omega_{3} \| \alpha_{i}\right|\left(\log \frac{\eta_{i}}{a}\right)^{q_{2}+p_{2}+\rho_{i}}}{\Gamma\left(q_{2}+p_{2}+\rho_{i}+1\right)}\right)\left(n_{1}\|x\|+n_{2}\|y\|+N_{2}\right) \\
& +\left(\frac{(T-a)^{q_{1}+p_{1}-1} \Gamma\left(q_{1}\right)}{|\Omega| \Gamma\left(q_{1}+p_{1}\right)}\left(\frac{\left|\lambda_{1}\right|\left|\Omega_{3}\right|\left|\sigma_{1}\right|\left(\tau_{1}-a\right)^{p_{1}}}{\Gamma\left(p_{1}+1\right)}+\sum_{j=1}^{n} \frac{\left|\lambda_{1}\right|\left|\Omega_{1}\right|\left|\beta_{j}\right|\left(\xi_{j}-a\right)^{p_{1}+\gamma_{j}}}{\Gamma\left(p_{1}+\gamma_{j}+1\right)}\right)\right. \\
& \left.+\frac{\left|\lambda_{1}\right|(T-a)^{p_{1}}}{\Gamma\left(p_{1}+1\right)}\right)\|x\|+\frac{(T-a)^{q_{1}+p_{1}-1} \Gamma\left(q_{1}\right)}{|\Omega| \Gamma\left(q_{1}+p_{1}\right)}\left(\sum_{i=1}^{m} \frac{\left|\lambda_{2}\right|\left|\Omega_{3}\right|\left|\alpha_{i}\right|\left(\log \frac{\eta_{i}}{a}\right)^{p_{2}+\rho_{i}}}{\Gamma\left(p_{2}+\rho_{i}+1\right)}\right. \\
& \left.+\frac{\left|\lambda_{2}\right|\left|\Omega_{1}\right|\left|\sigma_{2}\right|\left(\log \frac{\tau_{2}}{a}\right)^{p_{2}}}{\Gamma\left(p_{2}+1\right)}\right)\|y\| \\
& =\left(\frac{A_{1}}{|\Omega|}\left(\left|\sigma_{1}\right|\left|\Omega_{3}\right| A_{6}+\left|\Omega_{1}\right| A_{14}\right)+A_{4}\right)\left(m_{1}\|x\|+m_{2}\|y\|+N_{1}\right) \\
& +\frac{A_{1}}{|\Omega|}\left(\left|\sigma_{2}\right|\left|\Omega_{1}\right| A_{10}+\left|\Omega_{3}\right| A_{12}\right)\left(n_{1}\|x\|+n_{2}\|y\|+N_{2}\right) \\
& +\left(\frac{\left|\lambda_{1}\right| A_{1}}{|\Omega|}\left(\left|\sigma_{1}\right|\left|\Omega_{3}\right| A_{5}+\left|\Omega_{1}\right| A_{13}\right)+\left|\lambda_{1}\right| A_{3}\right)\|x\| \\
& +\frac{\left|\lambda_{2}\right| A_{1}}{|\Omega|}\left(\left|\sigma_{2}\right|\left|\Omega_{1}\right| A_{9}+\left|\Omega_{3}\right| A_{11}\right)\|y\|
\end{aligned}
$$




$$
\begin{aligned}
& =M_{1}\left(m_{1}\|x\|+m_{2}\|y\|+N_{1}\right)+M_{2}\left(n_{1}\|x\|+n_{2}\|y\|+N_{2}\right)+M_{3}\|x\|+M_{4}\|y\| \\
& =\left(m_{1} M_{1}+n_{1} M_{2}+M_{3}\right)\|x\|+\left(m_{2} M_{1}+n_{2} M_{2}+M_{4}\right)\|y\|+M_{1} N_{1}+M_{2} N_{2} \\
& \leq\left(\left(m_{1}+m_{2}\right) M_{1}+\left(n_{1}+n_{2}\right) M_{2}+M_{3}+M_{4}\right) r+M_{1} N_{1}+M_{2} N_{2} \\
& =B_{1} r+M_{1} N_{1}+M_{2} N_{2} \leq r .
\end{aligned}
$$

In the same way, we obtain

$$
\begin{aligned}
& \left|\mathcal{Q}_{2}(x, y)(t)\right| \\
& \leq\left(\frac{\left(\log \frac{T}{a}\right)^{q_{2}+p_{2}-1} \Gamma\left(q_{2}\right)}{|\Omega| \Gamma\left(q_{2}+p_{2}\right)}\left(\frac{\left|\Omega_{4}\right|\left|\sigma_{2}\right|\left(\log \frac{\tau_{2}}{a}\right)^{q_{2}+p_{2}}}{\Gamma\left(q_{2}+p_{2}+1\right)}+\sum_{i=1}^{m} \frac{\left|\Omega_{2}\right|\left|\alpha_{i}\right|\left(\log \frac{\eta_{i}}{a}\right)^{q_{2}+p_{2}+\rho_{i}}}{\Gamma\left(q_{2}+p_{2}+\rho_{i}+1\right)}\right)\right. \\
& \left.+\frac{\left(\log \frac{T}{a}\right)^{q_{2}+p_{2}}}{\Gamma\left(q_{2}+p_{2}+1\right)}\right)\left(n_{1}\|x\|+n_{2}\|y\|+N_{2}\right)+\frac{\left(\log \frac{T}{a}\right)^{q_{2}+p_{2}-1} \Gamma\left(q_{2}\right)}{|\Omega| \Gamma\left(q_{2}+p_{2}\right)} \\
& \times\left(\frac{\left|\Omega_{2}\right|\left|\sigma_{1}\right|\left(\tau_{1}-a\right)^{q_{1}+p_{1}}}{\Gamma\left(q_{1}+p_{1}+1\right)}+\sum_{j=1}^{n} \frac{\left|\Omega_{4}\right|\left|\beta_{j}\right|\left(\xi_{j}-a\right)^{q_{1}+p_{1}+\gamma_{j}}}{\Gamma\left(q_{1}+p_{1}+\gamma_{j}+1\right)}\right)\left(m_{1}\|x\|+m_{2}\|y\|+N_{1}\right) \\
& +\left(\frac{\left(\log \frac{T}{a}\right)^{q_{2}+p_{2}-1} \Gamma\left(q_{2}\right)}{|\Omega| \Gamma\left(q_{2}+p_{2}\right)}\left(\frac{\left|\lambda_{2}\right|\left|\Omega_{4}\right|\left|\sigma_{2}\right|\left(\log \frac{\tau_{2}}{a}\right)^{p_{2}}}{\Gamma\left(p_{2}+1\right)}+\sum_{i=1}^{m} \frac{\left|\lambda_{2}\right|\left|\Omega_{2}\right|\left|\alpha_{i}\right|\left(\log \frac{\eta_{i}}{a}\right)^{p_{2}+\rho_{i}}}{\Gamma\left(p_{2}+\rho_{i}+1\right)}\right)\right. \\
& \left.+\frac{\left|\lambda_{2}\right|\left(\log \frac{T}{a}\right)^{p_{2}}}{\Gamma\left(p_{2}+1\right)}\right)\|y\|+\frac{\left(\log \frac{T}{a}\right)^{q_{2}+p_{2}-1} \Gamma\left(q_{2}\right)}{|\Omega| \Gamma\left(q_{2}+p_{2}\right)}\left(\sum_{j=1}^{n} \frac{\left|\lambda_{1}\right|\left|\Omega_{4}\right|\left|\beta_{j}\right|\left(\xi_{j}-a\right)^{p_{1}+\gamma_{j}}}{\Gamma\left(p_{1}+\gamma_{j}+1\right)}\right. \\
& \left.+\frac{\left|\lambda_{1}\right|\left|\Omega_{2}\right|\left|\sigma_{1}\right|\left(\tau_{1}-a\right)^{p_{1}}}{\Gamma\left(p_{1}+1\right)}\right)\|x\| \\
& =\left(\frac{A_{2}}{|\Omega|}\left(\left|\Omega_{4}\right|\left|\sigma_{2}\right| A_{10}+\left|\Omega_{2}\right| A_{12}\right)+A_{8}\right)\left(n_{1}\|x\|+n_{2}\|y\|+N_{2}\right) \\
& +\frac{A_{2}}{|\Omega|}\left(\left|\Omega_{2}\right|\left|\sigma_{1}\right| A_{6}+\left|\Omega_{4}\right| A_{14}\right)\left(m_{1}\|x\|+m_{2}\|y\|+N_{1}\right) \\
& +\left(\left|\lambda_{2}\right| A_{7}+\frac{\left|\lambda_{2}\right| A_{2}}{|\Omega|}\left(\left|\Omega_{4}\right|\left|\sigma_{2}\right| A_{7}+\left|\Omega_{2}\right| A_{11}\right)\right)\|y\| \\
& +\frac{\left|\lambda_{1}\right| A_{2}}{|\Omega|}\left(\left|\Omega_{2}\right|\left|\sigma_{1}\right| A_{5}+\left|\Omega_{4}\right| A_{13}\right)\|x\| \\
& =M_{5}\left(n_{1}\|x\|+n_{2}\|y\|+N_{2}\right)+M_{6}\left(m_{1}\|x\|+m_{2}\|y\|+N_{1}\right)+M_{7}\|y\|+M_{8}\|x\| \\
& =\left(n_{1} M_{5}+m_{1} M_{6}+M_{8}\right)\|x\|+\left(n_{2} M_{5}+m_{2} M_{6}+M_{7}\right)\|y\|+M_{6} N_{1}+M_{5} N_{2} \\
& \leq\left(\left(m_{1}+m_{2}\right) M_{6}+\left(n_{1}+n_{2}\right) M_{5}+M_{7}+M_{8}\right) r+M_{6} N_{1}+M_{5} N_{2} \\
& =C_{1} r+M_{6} N_{1}+M_{5} N_{2} \leq r .
\end{aligned}
$$

Now for $\left(x_{2}, y_{2}\right),\left(x_{1}, y_{1}\right) \in X \times X$, and for any $t \in[a, T]$, we get

$$
\begin{aligned}
& \left|\mathcal{Q}_{1}\left(x_{2}, y_{2}\right)(t)-\mathcal{Q}_{1}\left(x_{1}, y_{1}\right)(t)\right| \\
& \leq \mathrm{RL}^{I_{1}+p_{1}}\left(\left|f\left(s, x_{2}(s), y_{2}(s)\right)-f\left(s, x_{1}(s), y_{1}(s)\right)\right|\right)(T)+\left|\lambda_{1}\right|_{\mathrm{RL}} I^{p_{1}}\left(\left|x_{2}(s)-x_{1}(s)\right|\right)(T) \\
& \quad+\frac{(T-a)^{q_{1}+p_{1}-1} \Gamma\left(q_{1}\right)}{|\Omega| \Gamma\left(q_{1}+p_{1}\right)}
\end{aligned}
$$




$$
\begin{aligned}
& \times\left(\left(\sum_{j=1}^{n}\left|\beta_{j}\right|_{\mathrm{RL}} I^{q_{1}+p_{1}+\gamma_{j}}\left(\left|f\left(s, x_{2}(s), y_{2}(s)\right)-f\left(s, x_{1}(s), y_{1}(s)\right)\right|\right)\left(\xi_{j}\right)\right.\right. \\
& +\left|\lambda_{1}\right| \sum_{j=1}^{n}\left|\beta_{j}\right|_{\mathrm{RL}} I^{p_{1}+\gamma_{j}}\left(\left|x_{2}(s)-x_{1}(s)\right|\right)\left(\xi_{j}\right)+\left|\lambda_{2}\right|\left|\sigma_{2}\right|_{\mathrm{H}} I^{p_{2}}\left(\left|y_{2}(s)-y_{1}(s)\right|\right)\left(\tau_{2}\right) \\
& \left.+\left|\sigma_{2}\right|_{\mathrm{H}} I^{q_{2}+p_{2}}\left(\left|g\left(s, x_{2}(s), y_{2}(s)\right)-g\left(s, x_{1}(s), y_{1}(s)\right)\right|\right)\left(\tau_{2}\right)\right)\left|\Omega_{1}\right| \\
& +\left(\sum_{i=1}^{m}\left|\alpha_{i}\right|_{\mathrm{H}} I^{q_{2}+p_{2}+\rho_{i}}\left(\left|g\left(s, x_{2}(s), y_{2}(s)\right)-g\left(s, x_{1}(s), y_{1}(s)\right)\right|\right)\left(\eta_{i}\right)\right. \\
& +\left|\lambda_{2}\right| \sum_{i=1}^{m}\left|\alpha_{i}\right|_{\mathrm{H}} I^{p_{2}+\rho_{i}}\left(\left|y_{2}(s)-y_{1}(s)\right|\right)\left(\eta_{i}\right)+\left|\lambda_{1}\right|\left|\sigma_{1}\right|_{\mathrm{RL}} I^{p_{1}}\left(\left|x_{2}(s)-x_{1}(s)\right|\right)\left(\tau_{1}\right) \\
& \left.\left.+\left|\sigma_{1}\right|_{\mathrm{RL}} I^{q_{1}+p_{1}}\left(\left|f\left(s, x_{2}(s), y_{2}(s)\right)-f\left(s, x_{1}(s), y_{1}(s)\right)\right|\right)\left(\tau_{1}\right)\right)\left|\Omega_{3}\right|\right) \\
& \leq\left(\frac{(T-a)^{q_{1}+p_{1}-1} \Gamma\left(q_{1}\right)}{|\Omega| \Gamma\left(q_{1}+p_{1}\right)}\left(\sum_{j=1}^{n} \frac{\left|\Omega_{1}\right|\left|\beta_{j}\right|\left(\xi_{j}-a\right)^{q_{1}+p_{1}+\gamma_{j}}}{\Gamma\left(q_{1}+p_{1}+\gamma_{j}+1\right)}+\frac{\left|\sigma_{1}\right|\left|\Omega_{3}\right|\left(\tau_{1}-a\right)^{q_{1}+p_{1}}}{\Gamma\left(q_{1}+p_{1}+1\right)}\right)\right. \\
& \left.+\frac{(T-a)^{q_{1}+p_{1}}}{\Gamma\left(q_{1}+p_{1}+1\right)}\right)\left(m_{1}\left\|x_{2}-x_{1}\right\|+m_{2}\left\|y_{2}-y_{1}\right\|\right)+\frac{(T-a)^{q_{1}+p_{1}-1} \Gamma\left(q_{1}\right)}{|\Omega| \Gamma\left(q_{1}+p_{1}\right)} \\
& \times\left(\frac{\left|\sigma_{2}\right|\left|\Omega_{1}\right|\left(\log \frac{\tau_{2}}{a}\right)^{q_{2}+p_{2}}}{\Gamma\left(q_{2}+p_{2}+1\right)}+\sum_{i=1}^{m} \frac{\left|\Omega_{3}\right|\left|\alpha_{i}\right|\left(\log \frac{\eta_{i}}{a}\right)^{q_{2}+p_{2}+\rho_{i}}}{\Gamma\left(q_{2}+p_{2}+\rho_{i}+1\right)}\right) \\
& \times\left(n_{1}\left\|x_{2}-x_{1}\right\|+n_{2}\left\|y_{2}-y_{1}\right\|\right) \\
& +\left(\frac{(T-a)^{q_{1}+p_{1}-1} \Gamma\left(q_{1}\right)}{|\Omega| \Gamma\left(q_{1}+p_{1}\right)}\left(\frac{\left|\lambda_{1}\right|\left|\sigma_{1}\right|\left|\Omega_{3}\right|\left(\tau_{1}-a\right)^{p_{1}}}{\Gamma\left(p_{1}+1\right)}+\sum_{j=1}^{n} \frac{\left|\lambda_{1}\right|\left|\Omega_{1}\right|\left|\beta_{j}\right|\left(\xi_{j}-a\right)^{p_{1}+\gamma_{j}}}{\Gamma\left(p_{1}+\gamma_{j}+1\right)}\right)\right. \\
& \left.+\frac{\left|\lambda_{1}\right|(T-a)^{p_{1}}}{\Gamma\left(p_{1}+1\right)}\right)\left\|x_{2}-x_{1}\right\|+\frac{(T-a)^{q_{1}+p_{1}-1} \Gamma\left(q_{1}\right)}{|\Omega| \Gamma\left(q_{1}+p_{1}\right)}\left(\sum_{i=1}^{m} \frac{\left|\lambda_{2}\right|\left|\Omega_{3} \| \alpha_{i}\right|\left(\log \frac{\eta_{i}}{a}\right)^{p_{2}+\rho_{i}}}{\Gamma\left(p_{2}+\rho_{i}+1\right)}\right. \\
& \left.+\frac{\left|\lambda_{2}\right|\left|\sigma_{2}\right|\left|\Omega_{1}\right|\left(\log \frac{\tau_{2}}{a}\right)^{p_{2}}}{\Gamma\left(p_{2}+1\right)}\right)\left\|y_{2}-y_{1}\right\| \\
& =\left(\frac{A_{1}}{|\Omega|}\left(\left|\sigma_{1}\right|\left|\Omega_{3}\right| A_{6}+\left|\Omega_{1}\right| A_{14}+A_{4}\right)\right)\left(m_{1}\left\|x_{2}-x_{1}\right\|+m_{2}\left\|y_{2}-y_{1}\right\|\right) \\
& +\left(\frac{A_{1}}{|\Omega|}\left(\left|\sigma_{2}\right|\left|\Omega_{1}\right| A_{10}+\left|\Omega_{3}\right| A_{12}\right)\right)\left(n_{1}\left\|x_{2}-x_{1}\right\|+n_{2}\left\|y_{2}-y_{1}\right\|\right) \\
& +\left(\frac{\left|\lambda_{1}\right| A_{1}}{|\Omega|}\left(\left|\sigma_{1}\right|\left|\Omega_{3}\right| A_{5}+\left|\Omega_{1}\right| A_{13}\right)+\left|\lambda_{1}\right| A_{3}\right)\left\|x_{2}-x_{1}\right\| \\
& +\left(\frac{\left|\lambda_{2}\right| A_{1}}{|\Omega|}\left(\left|\sigma_{2}\right|\left|\Omega_{1}\right| A_{9}+\left|\Omega_{3}\right| A_{11}\right)\right)\left\|y_{2}-y_{1}\right\| \\
& =M_{1}\left(m_{1}\left\|x_{2}-x_{1}\right\|+m_{2}\left\|y_{2}-y_{1}\right\|\right)+M_{2}\left(n_{1}\left\|x_{2}-x_{1}\right\|+n_{2}\left\|y_{2}-y_{1}\right\|\right) \\
& +M_{3}\left\|x_{2}-x_{1}\right\|+M_{4}\left\|y_{2}-y_{1}\right\| \\
& =\left(m_{1} M_{1}+n_{1} M_{2}+M_{3}\right)\left\|x_{2}-x_{1}\right\|+\left(m_{2} M_{1}+n_{2} M_{2}+M_{4}\right)\left\|y_{2}-y_{1}\right\|,
\end{aligned}
$$


and consequently we obtain

$$
\left\|\mathcal{Q}_{1}\left(x_{2}, y_{2}\right)(t)-\mathcal{Q}_{1}\left(x_{1}, y_{1}\right)\right\| \leq B_{1}\left(\left\|x_{2}-x_{1}\right\|+\left\|y_{2}-y_{1}\right\|\right) .
$$

Similarly,

$$
\left\|\mathcal{Q}_{2}\left(x_{2}, y_{2}\right)(t)-\mathcal{Q}_{2}\left(x_{1}, y_{1}\right)\right\| \leq C_{1}\left(\left\|x_{2}-x_{1}\right\|+\left\|y_{2}-y_{1}\right\|\right) .
$$

It follows from (3.1) and (3.2) that

$$
\left\|\mathcal{Q}\left(x_{2}, y_{2}\right)(t)-\mathcal{Q}\left(x_{1}, y_{1}\right)(t)\right\| \leq\left(B_{1}+C_{1}\right)\left(\left\|x_{2}-x_{1}\right\|+\left\|y_{2}-y_{1}\right\|\right) .
$$

Since $\left(B_{1}+C_{1}\right)<1$, therefore, $\mathcal{Q}$ is a contraction operator. So, by Banach's fixed point theorem, the operator $\mathcal{Q}$ has a unique fixed point, which is the unique solution of the problem (1.1). This completes the proof.

In the next result, we prove the existence of solutions for the problem (1.1) by applying the Leray-Schauder alternative.

Lemma 3.1 (Leray-Schauder alternative [37], p.4) Let G be a normed linear space and $F: G \rightarrow G$ be a completely continuous operator (i.e., a map that restricted to any bounded set in $G$ is compact). Let

$$
\mathcal{E}(F)=\{x \in G: x=\kappa F(x) \text { for some } 0<\kappa<1\} .
$$

Then either the set $\mathcal{E}(F)$ is unbounded, or $F$ has at least one fixed point.

For convenience, we set the constants

$$
\begin{aligned}
& E_{1}=\left(M_{1}+M_{6}\right) P_{1}+\left(M_{2}+M_{5}\right) R_{1}+M_{3}+M_{8}, \\
& E_{2}=\left(M_{1}+M_{6}\right) P_{2}+\left(M_{2}+M_{5}\right) R_{2}+M_{4}+M_{7}
\end{aligned}
$$

and

$$
E^{*}=\min \left\{1-E_{1}, 1-E_{2}\right\}
$$

Theorem 3.2 Assume that the functions $f, g:[a, T] \times \mathbb{R}^{2} \rightarrow \mathbb{R}$ are continuous functions and there exist real constants $P_{i}, R_{i} \geq 0(i=1,2)$ and $P_{0}>0, R_{0}>0$ such that $\forall x_{i} \in \mathbb{R}$ $(i=1,2)$ we have

$$
\begin{aligned}
& \left|f\left(t, x_{1}, x_{2}\right)\right| \leq P_{0}+P_{1}\left|x_{1}\right|+P_{2}\left|x_{2}\right|, \\
& \left|g\left(t, x_{1}, x_{2}\right)\right| \leq R_{0}+R_{1}\left|x_{1}\right|+R_{2}\left|x_{2}\right| .
\end{aligned}
$$

In addition it is assumed that

$$
E_{1}<1 \text { and } E_{2}<1 \text {. }
$$

Then there exists at least one solution for the boundary value problem (1.1). 
Proof First we show that the operator $\mathcal{Q}: X \times X \rightarrow X \times X$ is completely continuous. Note that $Q$ is continuous, since the functions $f$ and $g$ are continuous.

Let $U \subset X \times X$ be bounded. Then there exist positive constants $L_{1}$ and $L_{2}$ such that

$$
|f(t, x(t), y(t))| \leq L_{1}, \quad|g(t, x(t), y(t))| \leq L_{2}, \quad \forall(x, y) \in U,
$$

and a positive real number $r^{\prime}$ such that

$$
r^{\prime} \geq \max \left\{\frac{M_{1} L_{1}+M_{2} L_{2}}{1-\left(M_{3}+M_{4}\right)}, \frac{M_{5} L_{1}+M_{6} L_{2}}{1-\left(M_{7}+M_{8}\right)}\right\} .
$$

Then, for any $(x, y) \in U$ where $B_{r^{\prime}}=\left\{(x, y) \in X \times X:\|(x, y)\| \leq r^{\prime}\right\}$ and using Lemma 2.4, we have

$$
\begin{aligned}
&\left\|\mathcal{Q}_{1}(x, y)(t)\right\| \\
& \leq \mathrm{RL}^{I_{1}+p_{1}}|f(s, x(s), y(s))|(T)+\left|\lambda_{1}\right|_{\mathrm{RL}} I^{p_{1}}|x(s)|(T)+\frac{(T-a)^{q_{1}+p_{1}-1} \Gamma\left(q_{1}\right)}{|\Omega| \Gamma\left(q_{1}+p_{1}\right)} \\
& \quad \times\left(\left(\sum_{j=1}^{n}\left|\beta_{j}\right|_{\mathrm{RL}} I^{q_{1}+p_{1}+\gamma_{j}}|f(s, x(s), y(s))|\left(\xi_{j}\right)+\left|\lambda_{1}\right| \sum_{j=1}^{n}\left|\beta_{j}\right|_{\mathrm{RL}} I^{p_{1}+\gamma_{j}}|x(s)|\left(\xi_{j}\right)\right.\right. \\
&\left.+\left|\lambda_{2}\right|\left|\sigma_{2}\right|_{\mathrm{H}} I^{p_{2}}|y(s)|\left(\tau_{2}\right)+\left|\sigma_{2}\right|_{\mathrm{H}} I^{q_{2}+p_{2}}|g(s, x(s), y(s))|\left(\tau_{2}\right)\right)\left|\Omega_{1}\right| \\
&+\left(\sum_{i=1}^{m}\left|\alpha_{i}\right|_{\mathrm{H}} I^{q_{2}+p_{2}+\rho_{i} \mid}|g(s, x(s), y(s))|\left(\eta_{i}\right)+\left|\lambda_{2}\right| \sum_{i=1}^{m}\left|\alpha_{i}\right|_{\mathrm{H}} I^{p_{2}+\rho_{i}}|y(s)|\left(\eta_{i}\right)\right. \\
&\left.\left.+\left|\lambda_{1}\right|\left|\sigma_{1}\right|_{\mathrm{RL}} I^{p_{1}}|x(s)|\left(\tau_{1}\right)+\left|\sigma_{1}\right|_{\mathrm{RL}} I^{q_{1}+p_{1}}|f(s, x(s), y(s))|\left(\tau_{1}\right)\right)\left|\Omega_{3}\right|\right) \\
& \leq\left(\frac{A_{1}}{|\Omega|}\left(\left|\sigma_{1}\right|\left|\Omega_{3}\right| A_{6}+\left|\Omega_{1}\right| A_{14}\right)+A_{4}\right) L_{1}+\left(\frac{A_{1}}{|\Omega|}\left(\left|\sigma_{2}\right|\left|\Omega_{1}\right| A_{10}+\left|\Omega_{3}\right| A_{12}\right)\right) L_{2} \\
&+\left(\frac{\left|\lambda_{1}\right| A_{1}}{|\Omega|}\left(\left|\sigma_{1}\right|\left|\Omega_{3}\right| A_{5}+\left|\Omega_{1}\right| A_{13}\right)+\left|\lambda_{1}\right| A_{3}+\frac{\left|\lambda_{2}\right| A_{1}}{|\Omega|}\left(\left|\sigma_{2}\right|\left|\Omega_{1}\right| A_{9}+\left|\Omega_{3}\right| A_{11}\right)\right) r^{\prime} \\
&= M_{1} L_{1}+M_{2} L_{2}+\left(M_{3}+M_{4}\right) r^{\prime} .
\end{aligned}
$$

In the same way, we deduce that

$$
\begin{aligned}
&\left\|\mathcal{Q}_{2}(x, y)\right\| \\
& \leq\left(\frac{A_{2}}{|\Omega|}\left(\left|\sigma_{2}\right|\left|\Omega_{4}\right| A_{10}+\left|\Omega_{2}\right| A_{12}\right)+A_{8}\right) L_{2}+\left(\frac{A_{2}}{|\Omega|}\left(\left|\sigma_{1}\right|\left|\Omega_{2}\right| A_{6}+\left|\Omega_{4}\right| A_{14}\right)\right) L_{1} \\
&+\left(\frac{\left|\lambda_{2}\right| A_{2}}{|\Omega|}\left(\left|\sigma_{2}\right|\left|\Omega_{4}\right| A_{9}+\left|\Omega_{2}\right| A_{11}\right)+\left|\lambda_{2}\right| A_{7}+\frac{\left|\lambda_{1}\right| A_{2}}{|\Omega|}\left(\left|\sigma_{1}\right|\left|\Omega_{2}\right| A_{5}+\left|\Omega_{4}\right| A_{13}\right)\right) r^{\prime} \\
&= M_{6} L_{1}+M_{5} L_{2}+\left(M_{7}+M_{8}\right) r^{\prime} .
\end{aligned}
$$

Thus, it follows from the above inequalities that the operator $\mathcal{Q}$ is uniformly bounded. 
Next, we show that $\mathcal{Q}$ is equicontinuous. Let $t_{1}, t_{2} \in[a, T]$ with $t_{1}<t_{2}$. Then we have

$$
\begin{aligned}
\left|\mathcal{Q}_{1}(x, y)\left(t_{2}\right)-\mathcal{Q}_{1}(x, y)\left(t_{1}\right)\right| & \leq\left|{ }_{\mathrm{RL}} I^{q_{1}+p_{1}} f(s, x(s), y(s))\left(t_{2}\right)-\mathrm{RL} I^{q_{1}+p_{1}} f(s, x(s), y(s))\left(t_{1}\right)\right| \\
& +\left.\left|\lambda_{1}\right|\right|_{\mathrm{RL}} I^{p_{1}} x\left(t_{2}\right)-\mathrm{RL}^{I_{1}} x\left(t_{1}\right) \mid+\frac{\left|\left(t_{2}-a\right)^{q_{1}+p_{1}-1}-\left(t_{1}-a\right)^{q_{1}+p_{1}-1}\right| \Gamma\left(q_{1}\right)}{|\Omega| \Gamma\left(q_{1}+p_{1}\right)} \\
& \times\left(\left(\sum_{j=1}^{n}\left|\beta_{j}\right|_{\mathrm{RL}} I^{q_{1}+p_{1}+\gamma_{j}}|f(s, x(s), y(s))|\left(\xi_{j}\right)+\left|\lambda_{1}\right| \sum_{j=1}^{n}\left|\beta_{j}\right| \mathrm{RL} I^{p_{1}+\gamma_{j}}|x(s)|\left(\xi_{j}\right)\right.\right. \\
& \left.+\left|\sigma_{2}\right|_{\mathrm{H}} I^{q_{2}+p_{2}}|g(s, x(s), y(s))|\left(\tau_{2}\right)+\left|\lambda_{2}\right|\left|\sigma_{2}\right|_{\mathrm{H}} I^{p_{2}}|y(s)|\left(\tau_{2}\right)\right)\left|\Omega_{1}\right| \\
& +\left(\sum_{i=1}^{m}\left|\alpha_{i}\right|_{\mathrm{H}} I^{q_{2}+p_{2}+\rho_{i}}|g(s, x(s), y(s))|\left(\eta_{i}\right)+\left|\lambda_{2}\right| \sum_{i=1}^{m}\left|\alpha_{i}\right|_{\mathrm{H}} I^{p_{2}+\rho_{i}}|y(s)|\left(\eta_{i}\right)\right. \\
& \left.\left.+\left|\sigma_{1}\right|_{\mathrm{RL}} I^{q_{1}+p_{1}}|f(s, x(s), y(s))|\left(\tau_{1}\right)+\left|\lambda_{1}\right|\left|\sigma_{1}\right|_{\mathrm{RL}} I^{p_{1}}|x(s)|\left(\tau_{1}\right)\right)\left|\Omega_{3}\right|\right) \\
\leq & \frac{L_{1}}{\Gamma\left(q_{1}+p_{1}\right)}\left(\int_{a}^{t_{1}}\left[\left(t_{2}-a\right)^{q_{1}+p_{1}-1}-\left(t_{1}-a\right)^{q_{1}+p_{1}-1}\right] d s+\int_{t_{1}}^{t_{2}}\left(t_{2}-a\right)^{q_{1}+p_{1}-1} d s\right) \\
& +\frac{\left|\lambda_{1}\right| r^{\prime}}{\Gamma\left(p_{1}\right)}\left(\int_{a}^{t_{1}}\left[\left(t_{2}-a\right)^{p_{1}-1}-\left(t_{1}-a\right)^{p_{1}-1}\right] d s+\int_{t_{1}}^{t_{2}}\left(t_{2}-a\right)^{p_{1}-1} d s\right) \\
& +\frac{\left|\left(t_{2}-a\right)^{q_{1}+p_{1}-1}-\left(t_{1}-a\right)^{q_{1}+p_{1}-1}\right| \Gamma\left(q_{1}\right)}{|\Omega| \Gamma\left(q_{1}+p_{1}\right)}\left(\left(\left|\Omega_{1}\right| A_{14}+\left|\sigma_{1}\right|\left|\Omega_{3}\right| A_{6}\right) L_{1}\right. \\
& +\left(\left|\sigma_{2}\right|\left|\Omega_{1}\right| A_{10}+\left|\Omega_{3}\right| A_{12}\right) L_{2}+\left(\left|\lambda_{1}\right|\left|\Omega_{1}\right| A_{13}+\left|\lambda_{2}\right|\left|\sigma_{2}\right|\left|\Omega_{1}\right| A_{9}+\left|\lambda_{2}\right|\left|\Omega_{3}\right| A_{11}\right. \\
& \left.\left.+\left|\lambda_{1}\right|\left|\sigma_{1}\right|\left|\Omega_{3}\right| A_{5}\right) r^{\prime}\right) .
\end{aligned}
$$

Analogously, we can obtain

$$
\begin{aligned}
& \left|\mathcal{Q}_{2}(x, y)\left(t_{2}\right)-\mathcal{Q}_{2}(x, y)\left(t_{1}\right)\right| \\
& \leq\left|{ }_{\mathrm{H}} I^{q_{2}+p_{2}} g(s, x(s), y(s))\left(t_{2}\right)-{ }_{\mathrm{H}} I^{q_{2}+p_{2}} g(s, x(s), y(s))\left(t_{1}\right)\right| \\
& \quad+\left.\left|\lambda_{2}\right|\right|_{\mathrm{H}} I^{p_{2}} y\left(t_{2}\right)-{ }_{\mathrm{H}} I^{p_{2}} y\left(t_{1}\right) \mid+\frac{\left|\left(\log \frac{t_{2}}{a}\right)^{q_{2}+p_{2}-1}-\left(\log \frac{t_{1}}{a}\right)^{q_{2}+p_{2}-1}\right| \Gamma\left(q_{2}\right)}{|\Omega| \Gamma\left(q_{2}+p_{2}\right)} \\
& \quad \times\left(\left(\sum_{i=1}^{m}\left|\alpha_{i}\right|_{\mathrm{H}} I^{q_{2}+p_{2}+\rho_{i}}|g(s, x(s), y(s))|\left(\eta_{i}\right)+\left|\lambda_{2}\right| \sum_{i=1}^{m}\left|\alpha_{i}\right|_{\mathrm{H}} I^{p_{2}+\rho_{i}}|y(s)|\left(\eta_{i}\right)\right.\right. \\
& \left.\quad+\left|\sigma_{1}\right|_{\mathrm{RL}} I^{q_{1}+p_{1}}|f(s, x(s), y(s))|\left(\tau_{1}\right)+\left|\lambda_{1}\right|\left|\sigma_{1}\right|_{\mathrm{RL}} I^{p_{1}}|x(s)|\left(\tau_{1}\right)\right)\left|\Omega_{2}\right| \\
& \quad+\left(\sum_{j=1}^{n}\left|\beta_{j}\right|_{\mathrm{RL}} I^{q_{1}+p_{1}+\gamma_{j}}|f(s, x(s), y(s))|\left(\xi_{j}\right)+\left|\lambda_{1}\right| \sum_{j=1}^{n}\left|\beta_{j}\right|_{\mathrm{RL}} I^{p_{1}+\gamma_{j}}|x(s)|\left(\xi_{j}\right)\right. \\
& \left.\left.\quad+\left|\sigma_{2}\right|_{\mathrm{H}} I^{q_{2}+p_{2}}|g(s, x(s), y(s))|\left(\tau_{2}\right)+\left|\lambda_{2}\right|\left|\sigma_{2}\right|_{\mathrm{H}} I^{p_{2}}|y(s)|\left(\tau_{2}\right)\right)\left|\Omega_{4}\right|\right) \\
& \leq \frac{L_{2}}{\Gamma\left(q_{2}+p_{2}\right)}\left(\int_{a}^{t_{1}}\left(\left(\log \frac{t_{2}}{a}\right)^{q_{2}+p_{2}-1}-\left(\log \frac{t_{1}}{a}\right)^{q_{2}+p_{2}-1}\right) d s\right.
\end{aligned}
$$




$$
\begin{aligned}
& \left.+\int_{t_{1}}^{t_{2}}\left(\log \frac{t_{2}}{a}\right)^{q_{2}+p_{2}-1} d s\right) \\
& +\frac{\left|\lambda_{2}\right| r^{\prime}}{\Gamma\left(p_{2}\right)}\left(\int_{a}^{t_{1}}\left(\left(\log \frac{t_{2}}{a}\right)^{q_{2}+p_{2}-1}-\left(\log \frac{t_{1}}{a}\right)^{q_{2}+p_{2}-1}\right) d s+\int_{t_{1}}^{t_{2}}\left(\log \frac{t_{2}}{a}\right)^{q_{2}+p_{2}-1} d s\right) \\
& +\frac{\left|\left(\log \frac{t_{2}}{a}\right)^{q_{2}+p_{2}-1}-\left(\log \frac{t_{1}}{a}\right)^{q_{2}+p_{2}-1}\right| \Gamma\left(q_{2}\right)}{|\Omega| \Gamma\left(q_{2}+p_{2}\right)}\left(\left(\left|\sigma_{1}\right|\left|\Omega_{2}\right| A_{6}+\left|\Omega_{4}\right| A_{14}\right) L_{1}\right. \\
& +\left(\left|\Omega_{2}\right| A_{12}+\left|\sigma_{2}\right|\left|\Omega_{4}\right| A_{10}\right) L_{2}+\left(\left|\lambda_{1}\right|\left|\sigma_{1}\right|\left|\Omega_{2}\right| A_{5}+\left|\lambda_{1}\right|\left|\Omega_{4}\right| A_{13}+\left|\lambda_{2}\right|\left|\sigma_{2}\right|\left|\Omega_{4}\right| A_{9}\right. \\
& \left.\left.+\left|\lambda_{2}\right|\left|\Omega_{2}\right| A_{11}\right) r^{\prime}\right) .
\end{aligned}
$$

Therefore, the operator $\mathcal{Q}(x, y)$ is equicontinuous, and thus the operator $\mathcal{Q}(x, y)$ is completely continuous, by Arzelá-Ascoli theorem.

Finally, it will be verified that the set $\mathcal{E}=\{(x, y) \in X \times X \mid(x, y)=\kappa \mathcal{Q}(x, y), 0<\kappa<1\}$ is bounded. Let $(x, y) \in \mathcal{E}$, then $(x, y)=\kappa \mathcal{Q}(x, y)$. For any $t \in[a, T]$, we have

$$
x(t)=\kappa \mathcal{Q}_{1}(x, y)(t), \quad y(t)=\kappa \mathcal{Q}_{2}(x, y)(t) .
$$

Then

$$
\begin{aligned}
|x(t)| & \\
= & \left|\kappa \mathcal{Q}_{1}(x, y)(t)\right| \\
\leq & \left(P_{0}+P_{1}\|x\|+P_{2}\|y\|\right)_{\mathrm{RL}} I^{q_{1}+p_{1}}(1)(T)+\|x\|\left|\lambda_{1}\right|_{\mathrm{RL}} I^{p_{1}}(1)(T) \\
& +\frac{(T-a)^{q_{1}+p_{1}-1} \Gamma\left(q_{1}\right)}{|\Omega| \Gamma\left(q_{1}+p_{1}\right)}\left(\left(\left(P_{0}+P_{1}\|x\|+P_{2}\|y\|\right) \sum_{j=1}^{n}\left|\beta_{j}\right|_{\mathrm{RL}} I^{q_{1}+p_{1}+\gamma_{j}}(1)\left(\xi_{j}\right)\right.\right. \\
& +\|x\|\left|\lambda_{1}\right| \sum_{j=1}^{n}\left|\beta_{j}\right|_{\mathrm{RL}} I^{p_{1}+\gamma_{j}}(1)\left(\xi_{j}\right)+\left(R_{0}+R_{1}\|x\|+R_{2}\|y\|\right)\left|\sigma_{2}\right|_{\mathrm{H}} I^{q_{2}+p_{2}}(1)\left(\tau_{2}\right) \\
& \left.+\|y\|\left|\lambda_{2}\right|\left|\sigma_{2}\right|_{\mathrm{H}} I^{p_{2}}(1)\left(\tau_{2}\right)\right)\left|\Omega_{1}\right|+\left(\left(R_{0}+R_{1}\|x\|+R_{2}\|y\|\right) \sum_{i=1}^{m}\left|\alpha_{i}\right|_{\mathrm{H}} I^{q_{2}+p_{2}+\rho_{i}}(1)\left(\eta_{i}\right)\right. \\
& +\|y\|\left|\lambda_{2}\right| \sum_{i=1}^{m}\left|\alpha_{i}\right|_{\mathrm{H}} I^{p_{2}+\rho_{i}}(1)\left(\eta_{i}\right)+\left(P_{0}+P_{1}\|x\|+P_{2}\|y\|\right)\left|\sigma_{1}\right|_{\mathrm{RL}} I^{q_{1}+p_{1}}(1)\left(\tau_{1}\right) \\
& \left.\left.+\|x\|\left|\lambda_{1} \| \sigma_{1}\right|_{\mathrm{RL}} I^{p_{1}}(1)\left(\tau_{1}\right)\right)\left|\Omega_{3}\right|\right) \\
= & \left(P_{0}+P_{1}\|x\|+P_{2}\|y\|\right) M_{1}+\left(R_{0}+R_{1}\|x\|+R_{2}\|y\|\right) M_{2}+\|x\| M_{3}+\|y\| M_{4}
\end{aligned}
$$

and

$$
\begin{aligned}
|y(t)| & \\
= & \left|\kappa \mathcal{Q}_{2}(x, y)(t)\right| \\
\leq & \left(R_{0}+R_{1}\|x\|+R_{2}\|y\|\right)_{\mathrm{H}} I^{q_{2}+p_{2}}(1)(T)+\|y\|\left|\lambda_{2}\right|_{\mathrm{H}} I^{p_{2}}(1)(T) \\
& \quad+\frac{\left(\log \frac{T}{a}\right)^{q_{2}+p_{2}-1} \Gamma\left(q_{2}\right)}{|\Omega| \Gamma\left(q_{2}+p_{2}\right)}\left(\left(\left(R_{0}+R_{1}\|x\|+R_{2}\|y\|\right) \sum_{i=1}^{m}\left|\alpha_{i}\right|_{\mathrm{H}} I^{q_{2}+p_{2}+\rho_{i}}(1)\left(\eta_{i}\right)\right.\right.
\end{aligned}
$$




$$
\begin{aligned}
& +\|y\|\left|\lambda_{2}\right| \sum_{i=1}^{m}\left|\alpha_{i}\right|_{\mathrm{H}} I^{p_{2}+\rho_{i}}(1)\left(\eta_{i}\right)+\left(P_{0}+P_{1}\|x\|+P_{2}\|y\|\right)\left|\sigma_{1}\right|_{\mathrm{RL}} I^{q_{1}+p_{1}}(1)\left(\tau_{1}\right) \\
& \left.+\|x\|\left|\lambda_{1}\right|\left|\sigma_{1}\right|_{\mathrm{RL}} I^{p_{1}}(1)\left(\tau_{1}\right)\right)\left|\Omega_{2}\right|+\left(\left(P_{0}+P_{1}\|x\|+P_{2}\|y\|\right) \sum_{j=1}^{n}\left|\beta_{j}\right|_{\mathrm{RL}} I^{q_{1}+p_{1}+\gamma_{j}}(1)\left(\xi_{j}\right)\right. \\
& +\|x\|\left|\lambda_{1}\right| \sum_{j=1}^{n}\left|\beta_{j}\right|_{\mathrm{RL}} I^{p_{1}+\gamma_{j}}(1)\left(\xi_{j}\right)+\left(R_{0}+R_{1}\|x\|+R_{2}\|y\|\right)\left|\sigma_{2}\right|_{\mathrm{H}} I^{q_{2}+p_{2}}(1)\left(\tau_{2}\right) \\
& \left.\left.+\|y\|\left|\lambda_{2}\right|\left|\sigma_{2}\right|_{\mathrm{H}} I^{p_{2}}(1)\left(\tau_{2}\right)\right)\left|\Omega_{4}\right|\right) \\
& =\left(P_{0}+P_{1}\|x\|+P_{2}\|y\|\right) M_{6}+\left(R_{0}+R_{1}\|x\|+R_{2}\|y\|\right) M_{5}+\|x\| M_{8}+\|y\| M_{7} .
\end{aligned}
$$

Hence we have

$$
\|x\| \leq\left(P_{0}+P_{1}\|x\|+P_{2}\|y\|\right) M_{1}+\left(R_{0}+R_{1}\|x\|+R_{2}\|y\|\right) M_{2}+\|x\| M_{3}+\|y\| M_{4}
$$

and

$$
\|y\| \leq\left(P_{0}+P_{1}\|x\|+P_{2}\|y\|\right) M_{6}+\left(R_{0}+R_{1}\|x\|+R_{2}\|y\|\right) M_{5}+\|x\| M_{8}+\|y\| M_{7},
$$

which implies

$$
\begin{aligned}
\|x\|+\|y\| \leq & \left(M_{1}+M_{6}\right) P_{0}+\left(M_{2}+M_{5}\right) R_{0} \\
& +\left(\left(M_{1}+M_{6}\right) P_{1}+\left(M_{2}+M_{5}\right) R_{1}+M_{3}+M_{8}\right)\|x\| \\
& +\left(\left(M_{1}+M_{6}\right) P_{2}+\left(M_{2}+M_{5}\right) R_{2}+M_{4}+M_{7}\right)\|y\| .
\end{aligned}
$$

Consequently,

$$
\|(x, y)\| \leq \frac{\left(M_{1}+M_{6}\right) P_{0}+\left(M_{2}+M_{5}\right) R_{0}}{E^{*}}
$$

for any $t \in[a, T]$, where $E^{*}$ is defined by (3.3), which proves that $\mathcal{E}$ is bounded. Thus, by Lemma 3.1, the operator $\mathcal{Q}$ has at least one fixed point. Hence the boundary value problem (1.1) has at least one solution on $[a, T]$. The proof is complete.

\subsection{Examples}

In this section we present examples to illustrate our results.

Example 3.1 Consider the system of Langevin equations via the Riemann-Liouville and Hadamard fractional derivatives and fractional integral conditions:

$$
\left\{\begin{array}{l}
\mathrm{RL}^{2 / 5}\left(\mathrm{RL}^{3 / 4}+\frac{1}{7}\right) x(t)=\frac{|x| \sin ^{2}(2 \pi t)}{(9-t)^{2}}\left(\frac{|x|}{|x|+2}+1\right)+\frac{|y|}{(10-t)^{2}}-\frac{1}{2}, \\
{ }_{\mathrm{H}} D^{5 / 6}\left(\mathrm{H}_{\mathrm{H}} D^{3 / 7}-\frac{1}{11}\right) y(t)=\frac{|x|}{(10+t)^{2}}+\frac{\cos ^{2}(\pi t)}{(11-t)^{2}}\left(\frac{|y|}{|y|+3}+1\right)|y|+1, \\
x\left(\frac{1}{4}\right)=0, \quad \sqrt{2} x(1)=\frac{1}{2} \mathrm{H} I^{\sqrt{3}} y\left(\frac{1}{3}\right)-\frac{1}{3} \mathrm{H}^{4 / 5} y\left(\frac{3}{2}\right), \\
y\left(\frac{1}{4}\right)=0, \quad \frac{1}{2} y\left(\frac{3}{2}\right)=\frac{1}{6} \mathrm{RL} I^{\pi / 2} x\left(\frac{2}{5}\right)+\frac{1}{8} \mathrm{RL} I^{\sqrt{5}} x\left(\frac{5}{3}\right), \quad \frac{1}{4} \leq t \leq 2 .
\end{array}\right.
$$


Here $q_{1}=2 / 5, q_{2}=5 / 6, p_{1}=3 / 4, p_{2}=3 / 7, \lambda_{1}=1 / 7, \lambda_{2}=-1 / 11, n=2, m=2, a=1 / 4$, $T=2, \sigma_{1}=\sqrt{2}, \sigma_{2}=1 / 2, \tau_{1}=1, \tau_{2}=3 / 2, \eta_{1}=1 / 3, \eta_{2}=3 / 2, \xi_{1}=2 / 5, \xi_{2}=5 / 3, \alpha_{1}=$ $1 / 2, \alpha_{2}=-1 / 3, \beta_{1}=1 / 6, \beta_{2}=1 / 8, \rho_{1}=\sqrt{3}, \rho_{2}=4 / 5, \gamma_{1}=\pi / 2, \gamma_{2}=\sqrt{5}$, and $f(t, x, y)=$ $\left(\left(\sin ^{2}(2 \pi t)\right) /\left((9-t)^{2}\right)\right)(|x| /(|x|+2)+1)|x|+\left(|y| /\left((10-t)^{2}\right)\right)-(1 / 2)$ and $g(t, x, y)=(|x| /((10+$ $\left.\left.t)^{2}\right)\right)+\left(\cos ^{2}(\pi t)\right) /\left((11-t)^{2}\right)(|y| /(|y|+3)+1)|y|+1$. Since

$$
\left|f\left(t, x_{1}, y_{1}\right)-f\left(t, x_{2}, y_{2}\right)\right| \leq \frac{24}{1,225}\left|x_{1}-x_{2}\right|+\frac{16}{1,521}\left|y_{1}-y_{2}\right|
$$

and

$$
\left|g\left(t, x_{1}, y_{1}\right)-g\left(t, x_{2}, y_{2}\right)\right| \leq \frac{16}{1,681}\left|x_{1}-x_{2}\right|+\frac{64}{5,547}\left|y_{1}-y_{2}\right| .
$$

By using the Maple program, we can find that

$$
\Omega=\Omega_{1} \Omega_{2}-\Omega_{3} \Omega_{4} \simeq-2.490241444 \neq 0 .
$$

Then the assumptions of Theorem 3.1 are satisfied with $m_{1}=24 / 1,225, m_{2}=16 / 1,521, n_{1}=$ $16 / 1,681, n_{2}=64 / 5,547, M_{1} \simeq 2.584592457, M_{2} \simeq 1.020410144, M_{3} \simeq 0.3762005941$, $M_{4} \simeq 0.1378778032, M_{5} \simeq 3.202563776, M_{6} \simeq 0.2101813160, M_{7} \simeq 0.2770092726$, $M_{8} \simeq 0.026253698333$, and

$$
\begin{aligned}
& B_{1}=\left(m_{1}+m_{2}\right) M_{1}+\left(n_{1}+n_{2}\right) M_{2}+M_{3}+M_{4} \approx 0.6133893264, \\
& C_{1}=\left(m_{1}+m_{2}\right) M_{6}+\left(n_{1}+n_{2}\right) M_{5}+M_{7}+M_{8} \approx 0.3770246907 .
\end{aligned}
$$

Therefore, we get

$$
B_{1}+C_{1} \simeq 0.9904140171<1 \text {. }
$$

Hence, by Theorem 3.1, the problem (3.4) has a unique solution on $[1 / 4,2]$.

Example 3.2 Consider the system of Langevin equations via the Riemann-Liouville and Hadamard fractional derivatives and fractional integral conditions:

$$
\left\{\begin{array}{l}
\left.\mathrm{RL}^{2 / 3} D_{\mathrm{RL}} D^{8 / 9}-\frac{1}{9}\right) x(t)=\frac{\sqrt{2}}{2}+\frac{|x| \pi^{2} \cos ^{2}(2 \pi t)}{8(9 \pi-t)^{2}}+\frac{5 \pi^{2}|y|}{4(9 \pi-t)^{2}}\left(\frac{|y|}{|y|+4}+1\right), \\
{ }_{\mathrm{H}} D^{7 / 8}\left({ }_{\mathrm{H}} D^{9 / 10}-\frac{1}{24}\right) y(t)=\frac{\sqrt{3}}{2}+\frac{\pi^{2}|x|}{9(5 \pi-t)^{2}}\left(\frac{|x|}{|x|+2}+1\right)+\frac{5 \pi^{2} \sin ^{2} y(t)}{2(8 \pi-t)^{2}}, \\
x\left(\frac{\pi}{2}\right)=0, \quad \frac{1}{5} x(\pi)=\sqrt{2}{ }_{\mathrm{H}} I^{1 / 3} y(\pi)-\frac{1}{2}{ }_{\mathrm{H}} I^{1 / 4} y\left(\frac{\pi}{2}\right)+\frac{4}{5} I_{\mathrm{H}} I^{1 / 5} y(2 \pi), \\
y\left(\frac{\pi}{2}\right)=0, \quad \frac{\sqrt{2}}{2} y\left(\frac{3 \pi}{2}\right)=3_{\mathrm{RL}} I^{1 / 2} x\left(\frac{3 \pi}{2}\right)-\mathrm{RL} I^{1 / 3} x(\pi), \quad \frac{\pi}{2} \leq t \leq 2 \pi .
\end{array}\right.
$$

Here $q_{1}=2 / 3, q_{2}=7 / 8, p_{1}=8 / 9, p_{2}=9 / 10, \lambda_{1}=-1 / 9, \lambda_{2}=-1 / 24, n=2, m=3, a=\pi / 2$, $T=2 \pi, \sigma_{1}=1 / 5, \sigma_{2}=\sqrt{2} / 2, \tau_{1}=\pi, \tau_{2}=3 \pi / 2, \eta_{1}=\pi, \eta_{2}=\pi / 2, \eta_{3}=2 \pi, \xi_{1}=3 \pi / 2, \xi_{2}=\pi$, $\alpha_{1}=\sqrt{2}, \alpha_{2}=-1 / 2, \alpha_{3}=4 / 5, \beta_{1}=3, \beta_{2}=-1, \rho_{1}=1 / 3, \rho_{2}=1 / 4, \rho_{3}=1 / 5, \gamma_{1}=1 / 2, \gamma_{2}=1 / 3$, and $f(t, x, y)=(\sqrt{2} / 2)+\left(|x| \pi^{2} \cos ^{2}(2 \pi t)\right) /\left(8(9 \pi-t)^{2}\right)+\left(5 \pi^{2}|y| /\left(4(9 \pi-t)^{2}\right)\right)(|y| /(|y|+4)+1)$ and $g(t, x, y)=(\sqrt{3} / 2)+\left(\pi^{2}|x| /\left(9(5 \pi-t)^{2}\right)\right)(|x| /(|x|+2)+1)+\left(5 \pi^{2} \sin ^{2} y(t)\right) /\left(2(8 \pi-t)^{2}\right)$. We have

$$
\left|f\left(t, x_{1}, x_{2}\right)\right| \leq \frac{\sqrt{2}}{2}+\frac{1}{578}\left|x_{1}\right|+\frac{5}{289}\left|x_{2}\right|
$$


and

$$
\left|g\left(t, x_{1}, x_{2}\right)\right| \leq \frac{\sqrt{3}}{2}+\frac{4}{729}\left|x_{1}\right|+\frac{2}{45}\left|x_{2}\right| .
$$

By using the Maple program, we can find that

$$
\Omega=\Omega_{1} \Omega_{2}-\Omega_{3} \Omega_{4} \simeq 24.06826232 \neq 0 .
$$

Then the assumptions of Theorem 3.2 are satisfied with $P_{0}=\sqrt{2} / 2, P_{1}=1 / 578, P_{2}=$ $5 / 289, R_{0}=\sqrt{3} / 2, R_{1}=4 / 729, R_{2}=2 / 45, M_{1} \simeq 6.576589776, M_{2} \simeq 0.3108591355, M_{3} \simeq$ 0.9554741131, $M_{4} \simeq 0.05738592491, M_{5} \simeq 0.8374143237, M_{6} \simeq 0.6069399031, M_{7} \simeq$ $0.1145557760, M_{8} \simeq 0.02300665416$, and

$$
\begin{aligned}
& E_{1}=\left(M_{1}+M_{6}\right) P_{1}+\left(M_{2}+M_{5}\right) R_{1}+M_{3}+M_{8} \simeq 0.9972095596<1, \\
& E_{2}=\left(M_{1}+M_{6}\right) P_{2}+\left(M_{2}+M_{5}\right) R_{2}+M_{4}+M_{7} \simeq 0.3472585973<1
\end{aligned}
$$

and

$$
E^{*}=\min \left\{1-E_{1}, 1-E_{2}\right\}=\min \{0.0027904404,0.6527414027\}=0.0027904404 .
$$

Thus all the conditions of Theorem 3.2 hold true and consequently as regards the conclusion of Theorem 3.2, for the problem (3.5) there exists at least one solution on $[\pi / 2,2 \pi]$.

\section{Uncoupled integral boundary conditions case}

In this section we consider the following system:

$$
\left\{\begin{array}{l}
\left.\mathrm{RL}^{\mathrm{R}_{1}} D_{\mathrm{RL}} D^{p_{1}}+\lambda_{1}\right) x(t)=f(t, x(t), y(t)), \quad a \leq t \leq T, \\
\mathrm{H}^{q_{2}}\left({ }_{\mathrm{H}} D^{p_{2}}+\lambda_{2}\right) y(t)=g(t, x(t), y(t)), \quad a \leq t \leq T, \\
x(a)=0, \quad \sigma_{1} x\left(\tau_{1}\right)=\sum_{i=1}^{m} \alpha_{i \mathrm{RL}} I^{\rho_{i}} x\left(\eta_{i}\right), \\
y(a)=0, \quad \sigma_{2} y\left(\tau_{2}\right)=\sum_{j=1}^{n} \beta_{j \mathrm{H}} I^{\gamma_{j}} y\left(\xi_{j}\right) .
\end{array}\right.
$$

Definition 4.1 A $(x, y) \in X \times X$ is said to be a solution of the system (4.1) if $(x, y)$ satisfies the system ${ }_{\mathrm{RL}} D^{q_{1}}\left({ }_{\mathrm{RL}} D^{p_{1}}+\lambda_{1}\right) x(t)=f(t, x(t), y(t)),{ }_{\mathrm{H}} D^{q_{2}}\left({ }_{\mathrm{H}} D^{p_{2}}+\lambda_{2}\right) y(t)=g(t, x(t), y(t))$, on $[a, T]$, and the conditions $x(a)=0, \sigma_{1} x\left(\tau_{1}\right)=\sum_{i=1}^{m} \alpha_{i \mathrm{RL}} I^{\rho_{i}} x\left(\eta_{i}\right), y(a)=0, \sigma_{2} y\left(\tau_{2}\right)=$ $\sum_{j=1}^{n} \beta_{j \mathrm{H}} I^{\gamma_{j}} y\left(\xi_{j}\right)$.

Lemma 4.1 (Auxiliary lemma) For $h \in C([a, T], \mathbb{R})$, the problem

$$
\left\{\begin{array}{l}
\mathrm{RL}^{q_{1}}\left(\mathrm{RL} D^{p_{1}}+\lambda_{1}\right) x(t)=h(t), \quad 0<q_{1}, p_{1} \leq 1,1<q_{1}+p_{1} \leq 2, \\
x(a)=0, \quad \sigma_{1} x\left(\tau_{1}\right)=\sum_{i=1}^{m} \alpha_{i \mathrm{RL}} I^{p_{i}} x\left(\eta_{i}\right), \quad t \in[a, T],
\end{array}\right.
$$

has a solution if and only if the equation

$$
\begin{aligned}
x(t)= & \mathrm{RL}^{q_{1}+p_{1}} h(t)-\lambda_{1 \mathrm{RL}} I^{p_{1}} x(t)-\frac{(t-a)^{q_{1}+p_{1}-1} \Gamma\left(q_{1}\right)}{\Psi_{1} \Gamma\left(q_{1}+p_{1}\right)}\left(\sum_{i=1}^{m} \alpha_{i \mathrm{RL}} I^{q_{1}+p_{1}+\rho_{i}} h\left(\eta_{i}\right)\right. \\
& \left.-\lambda_{1} \sum_{i=1}^{m} \alpha_{i \mathrm{RL}} I^{p_{1}+\rho_{i}} x\left(\eta_{i}\right)+\lambda_{1} \sigma_{1 \mathrm{RL}} I^{p_{1}} x\left(\tau_{1}\right)-\sigma_{1 \mathrm{RL}} I^{q_{1}+p_{1}} h\left(\tau_{1}\right)\right)
\end{aligned}
$$


has a solution, where

$$
\Psi_{1}:=\sum_{i=1}^{m} \frac{\alpha_{i}\left(\eta_{i}-a\right)^{q_{1}+p_{1}+\rho_{i}-1} \Gamma\left(q_{1}\right)}{\Gamma\left(q_{1}+p_{1}+\rho_{i}\right)}-\frac{\sigma_{1} \Gamma\left(q_{1}\right)\left(\tau_{1}-a\right)^{q_{1}+p_{1}-1}}{\Gamma\left(q_{1}+p_{1}\right)} \neq 0 .
$$

\subsection{Existence results for uncoupled case}

In view of Lemma 4.1, we define an operator $\mathcal{K}: X \times X \rightarrow X \times X$ by

$$
\mathcal{K}(x, y)(t)=\left(\begin{array}{l}
\mathcal{K}_{1}(x, y)(t) \\
\mathcal{K}_{2}(x, y)(t)
\end{array}\right)
$$

where

$$
\begin{aligned}
\mathcal{K}_{1}(x, y)(t)= & \mathrm{RL}^{I_{1}+p_{1}} f(s, x(s), y(s))(t)-\lambda_{1 \mathrm{RL}} I^{p_{1}} x(t)-\frac{(t-a)^{q_{1}+p_{1}-1} \Gamma\left(q_{1}\right)}{\Psi_{1} \Gamma\left(q_{1}+p_{1}\right)} \\
& \times\left(\sum_{i=1}^{m} \alpha_{i \mathrm{RL}} I^{q_{1}+p_{1}+\rho_{i}} f(s, x(s), y(s))\left(\eta_{i}\right)-\lambda_{1} \sum_{i=1}^{m} \alpha_{i \mathrm{RL}} I^{p_{1}+\rho_{i}} x\left(\eta_{i}\right)\right. \\
& \left.+\lambda_{1} \sigma_{1 \mathrm{RL}} I^{p_{1}} x\left(\tau_{1}\right)-\sigma_{1 \mathrm{RL}} I^{q_{1}+p_{1}} f(s, x(s), y(s))\left(\tau_{1}\right)\right)
\end{aligned}
$$

and

$$
\begin{aligned}
\mathcal{K}_{2}(x, y)(t)= & { }_{\mathrm{H}} I^{q_{2}+p_{2}} g(s, x(s), y(s))(t)-\lambda_{2 \mathrm{H}} I^{p_{2}} y(t)-\frac{\left(\log \frac{t}{a}\right)^{q_{2}+p_{2}-1} \Gamma\left(q_{2}\right)}{\Psi_{2} \Gamma\left(q_{2}+p_{2}\right)} \\
& \times\left(\sum_{j=1}^{n} \beta_{j \mathrm{H}} I^{q_{2}+p_{2}+\gamma_{j}} g(s, x(s), y(s))\left(\xi_{j}\right)-\lambda_{2} \sum_{j=1}^{n} \beta_{j \mathrm{H}} I^{p_{2}+\gamma_{j}} y\left(\xi_{j}\right)\right. \\
& \left.+\lambda_{2} \sigma_{2 \mathrm{H}} I^{p_{2}} y\left(\tau_{2}\right)-\sigma_{2 \mathrm{H}} I^{q_{2}+p_{2}} g(s, x(s), y(s))\left(\tau_{2}\right)\right)
\end{aligned}
$$

where

$$
\Psi_{2}:=\sum_{j=1}^{n} \frac{\beta_{j}\left(\log \frac{\xi_{j}}{a}\right)^{q_{2}+p_{2}+\gamma_{j}-1} \Gamma\left(q_{2}\right)}{\Gamma\left(q_{2}+p_{2}+\gamma_{j}\right)}-\frac{\sigma_{2} \Gamma\left(q_{2}\right)\left(\log \frac{\tau_{2}}{a}\right)^{q_{2}+p_{2}-1}}{\Gamma\left(q_{2}+p_{2}\right)} \neq 0 .
$$

For the sake of convenience, we set

$$
\begin{array}{ll}
A_{15}=\sum_{i=1}^{m} \frac{\left|\alpha_{i}\right|\left(\eta_{i}-a\right)^{p_{1}+\rho_{i}}}{\Gamma\left(p_{1}+\rho_{i}+1\right)}, & A_{16}=\sum_{i=1}^{m} \frac{\left|\alpha_{i}\right|\left(\eta_{i}-a\right)^{q_{1}+p_{1}+\rho_{i}}}{\Gamma\left(q_{1}+p_{1}+\rho_{i}+1\right)}, \\
A_{17}=\sum_{j=1}^{n} \frac{\left|\beta_{j}\right|\left(\log \frac{\xi_{j}}{a}\right)^{p_{2}+\gamma_{j}}}{\Gamma\left(p_{2}+\gamma_{j}+1\right)}, & A_{18}=\sum_{j=1}^{n} \frac{\left|\beta_{j}\right|\left(\log \frac{\xi_{j}}{a}\right)^{q_{2}+p_{2}+\gamma_{j}}}{\Gamma\left(q_{2}+p_{2}+\gamma_{j}+1\right)}
\end{array}
$$

and

$$
\begin{array}{ll}
M_{9}=\frac{A_{1}}{\left|\Psi_{1}\right|}\left(\left|\sigma_{1}\right| A_{6}+A_{16}\right)+A_{4}, & M_{10}=\frac{\left|\lambda_{1}\right| A_{1}}{\left|\Psi_{1}\right|}\left(\left|\sigma_{1}\right| A_{5}+A_{15}\right)+\left|\lambda_{1}\right| A_{3}, \\
M_{11}=\frac{A_{2}}{\left|\Psi_{2}\right|}\left(\left|\sigma_{2}\right| A_{10}+A_{18}\right)+A_{8}, \quad M_{12}=\frac{\left|\lambda_{2}\right| A_{2}}{\left|\Psi_{2}\right|}\left(\left|\sigma_{2}\right| A_{9}+A_{17}\right)+\left|\lambda_{2}\right| A_{7} .
\end{array}
$$


Now we state the existence and uniqueness result for the problem (4.1). We do not provide the proof of this result because it is similar to that of Theorem 3.1.

Theorem 4.1 Assume that $f, g:[0, T] \times \mathbb{R}^{2} \rightarrow \mathbb{R}$ are continuous functions and there exist constants $\bar{m}_{i}, \bar{n}_{i}, i=1,2$ such that for all $t \in[a, T]$ and $x_{i}, y_{i} \in \mathbb{R}, i=1,2$,

$$
\left|f\left(t, x_{1}, y_{1}\right)-f\left(t, x_{2}, y_{2}\right)\right| \leq \bar{m}_{1}\left|x_{1}-x_{2}\right|+\bar{m}_{2}\left|y_{1}-y_{2}\right|
$$

and

$$
\left|g\left(t, x_{1}, y_{1}\right)-g\left(t, x_{2}, y_{2}\right)\right| \leq \bar{n}_{1}\left|x_{1}-x_{2}\right|+\bar{n}_{2}\left|y_{1}-y_{2}\right| .
$$

\section{Assume, in addition}

$$
\delta_{1}+\delta_{2}<1
$$

where

$$
\begin{aligned}
& \delta_{1}=\bar{m}_{1} M_{9}+\bar{m}_{2} M_{9}+M_{10}, \\
& \delta_{2}=\bar{n}_{1} M_{11}+\bar{n}_{2} M_{11}+M_{12} .
\end{aligned}
$$

Then the boundary value problem (4.1) has a unique solution.

Example 4.1 Consider the system of Langevin equations via the Riemann-Liouville and Hadamard fractional derivatives and fractional integral conditions:

$$
\left\{\begin{array}{l}
\left.\mathrm{RL}^{1 / 2} D_{\mathrm{RL}} D^{4 / 7}-\frac{1}{36}\right) x(t)=\frac{|x|}{5(t+1)^{2}}\left(\frac{|x|}{|x|+3}+1\right)+\frac{\sin y(t)}{4(t+3)^{2}}-2, \\
{ }_{\mathrm{H}} D^{7 / 9}\left({ }_{\mathrm{H}} D^{1 / 3}+\frac{1}{25}\right) y(t)=\frac{|x| \sin ^{2}(\pi t)}{(5+t))^{2}}+\frac{|y| \sin ^{2}(3 \pi t)}{8(2 t))^{2}}\left(\frac{|y|}{|y|+3}+1\right)+\frac{1}{3}, \\
x\left(\frac{1}{10}\right)=0, \quad \frac{1}{5} x\left(\frac{1}{4}\right)=\frac{1}{2} \mathrm{RL} I^{1 / 4} x\left(\frac{1}{5}\right)-\frac{\sqrt{2}}{3} \mathrm{RL} I^{1 / 2} y\left(\frac{1}{6}\right), \\
y\left(\frac{1}{10}\right)=0, \quad \frac{1}{3} y\left(\frac{1}{3}\right)=\frac{\sqrt{3}}{6}{ }_{\mathrm{H}} I^{\sqrt{2} / 5} y\left(\frac{1}{8}\right)-\frac{1}{3} \mathrm{H}^{3 / 5} y\left(\frac{1}{9}\right), \quad \frac{1}{10} \leq t \leq \frac{1}{2} .
\end{array}\right.
$$

Here $q_{1}=1 / 2, q_{2}=7 / 9, p_{1}=4 / 7, p_{2}=1 / 3, \lambda_{1}=-1 / 36, \lambda_{2}=1 / 25, n=2, m=2, a=1 / 10$, $T=1 / 2, \sigma_{1}=1 / 5, \sigma_{2}=1 / 3, \tau_{1}=1 / 4, \tau_{2}=1 / 3, \eta_{1}=1 / 5, \eta_{2}=1 / 6, \xi_{1}=1 / 8, \xi_{2}=1 / 9, \alpha_{1}=1 / 2$, $\alpha_{2}=-\sqrt{2} / 3, \beta_{1}=\sqrt{3} / 6, \beta_{2}=-1 / 3, \rho_{1}=1 / 4, \rho_{2}=1 / 2, \gamma_{1}=\sqrt{2} / 5, \gamma_{2}=3 / 5$, and $f(t, x, y)=$ $\left(|x| / 5(t+1)^{2}\right)(|x| /(|x|+3)+1)+\left((\sin y(t)) / 4(t+3)^{2}\right)-2$ and $g(t, x, y)=\left(|x| \sin ^{2}(\pi t)\right) /((5+$ $\left.t)^{2}\right)+\left(|y| \sin ^{2}(3 \pi t)\right) /\left(8(2+t)^{2}\right)(|y| /(|y|+3)+1)+(1 / 3)$. We have

$$
\left|f\left(t, x_{1}, x_{2}\right)-f\left(t, y_{1}, y_{2}\right)\right| \leq \frac{16}{135}\left|x_{1}-x_{2}\right|+\frac{1}{49}\left|y_{1}-y_{2}\right|
$$

and

$$
\left|g\left(t, x_{1}, x_{2}\right)-g\left(t, y_{1}, y_{2}\right)\right| \leq \frac{4}{121}\left|x_{1}-x_{2}\right|+\frac{2}{75}\left|y_{1}-y_{2}\right| .
$$

Then the assumptions of Theorem 3.1 are satisfied with $\bar{m}_{1}=16 / 135, \bar{m}_{2}=1 / 49, \bar{n}_{1}=4 / 121$, and $\bar{n}_{2}=2 / 75$. By using the Maple program, we can find that

$$
\Psi_{1}=\sum_{i=1}^{m} \frac{\alpha_{i} \Gamma\left(q_{1}\right)\left(\eta_{i}-a\right)^{q_{1}+p_{1}+\rho_{i}-1}}{\Gamma\left(q_{1}+p_{1}+\rho_{i}\right)}-\frac{\sigma_{1} \Gamma\left(q_{1}\right)\left(\tau_{1}-a\right)^{q_{1}+p_{1}-1}}{\Gamma\left(q_{1}+p_{1}\right)} \simeq-0.0482501053 \neq 0,
$$


and $M_{9} \simeq 2.160475289, M_{10} \simeq 0.1985361506, M_{11} \simeq 3.472362774, M_{12} \simeq 0.1522555714$ with

$$
\begin{aligned}
& \delta_{1}=\bar{m}_{1} M_{9}+\bar{m}_{2} M_{9}+M_{10} \simeq 0.4586442394 \\
& \delta_{2}=\bar{n}_{1} M_{11}+\bar{n}_{2} M_{11}+M_{12} \simeq 0.3596407640 .
\end{aligned}
$$

Therefore, we get

$$
\delta_{1}+\delta_{2} \simeq 0.8182850034<1
$$

Hence, by Theorem 4.1, the problem (4.6) has a unique solution on [1/10,1/2].

The second result, dealing with the existence of solutions for the problem (4.1), is analogous to Theorem 3.2 and is given below.

Theorem 4.2 Assume that there exist real constants $u_{i}, v_{i} \geq 0(i=1,2)$ and $u_{0}>0, v_{0}>0$ such that $\forall x_{i} \in \mathbb{R}(i=1,2)$ we have

$$
\begin{gathered}
\left|f\left(t, x_{1}, x_{2}\right)\right| \leq u_{0}+u_{1}\left|x_{1}\right|+u_{2}\left|x_{2}\right| \\
\left|g\left(t, x_{1}, x_{2}\right)\right| \leq v_{0}+v_{1}\left|x_{1}\right|+v_{2}\left|x_{2}\right| .
\end{gathered}
$$

In addition it is assumed that

$$
l_{1}<1 \text { and } l_{2}<1 \text {, }
$$

where

$$
l_{1}=u_{1} M_{9}+v_{1} M_{11}+M_{10} \quad \text { and } \quad l_{2}=u_{2} M_{9}+v_{2} M_{11}+M_{12} .
$$

Then the boundary value problem (4.1) has at least one solution.

Proof Setting

$$
l_{0}=\min \left\{1-l_{1}, 1-l_{2}\right\}
$$

the proof is similar to that of Theorem 3.2. So we omit it.

Example 4.2 Consider the system of Langevin equations via the Riemann-Liouville and Hadamard fractional derivatives and fractional integral conditions:

$$
\left\{\begin{array}{l}
\mathrm{RL} D^{4 / 11}\left({ }_{\mathrm{RL}} D^{9 / 11}+\frac{1}{13}\right) x(t)=\frac{1}{2}+\frac{|x| \sin (\pi t)}{(1+t)^{2}}+\frac{|y|}{20(1+t)^{2}}\left(\frac{|y|}{|y|+4}+1\right), \\
{ }_{\mathrm{H}} D^{5 / 8}\left({ }_{\mathrm{H}} D^{7 / 8}+\frac{1}{15}\right) y(t)=\frac{1}{3}+\frac{|x|}{(4+t)^{2}} \cdot\left(\frac{|x|}{|x|+5}+1\right)+\frac{\cos ^{9} y(t)}{9(2+t)^{2}}, \\
x\left(\frac{\sqrt{2}}{10}\right)=0, \quad \frac{1}{\sqrt{5}} x\left(\frac{\sqrt{2}}{2}\right)=\frac{1}{5} \mathrm{RL} I^{\sqrt{3} / 2} x\left(\frac{\sqrt{2}}{9}\right)-\frac{1}{7} \mathrm{RL} I^{7 / 11} y\left(\frac{\sqrt{2}}{5}\right), \\
y\left(\frac{\sqrt{2}}{10}\right)=0, \quad \frac{1}{\sqrt{7}} y\left(\frac{\sqrt{2}}{3}\right)=\frac{2}{9} I^{4 / 9} y\left(\frac{\sqrt{2}}{4}\right)-\frac{1}{6} \mathrm{H}^{1 / 9} y\left(\frac{\sqrt{2}}{7}\right), \quad \frac{\sqrt{2}}{10} \leq t \leq \sqrt{2} .
\end{array}\right.
$$


Here $q_{1}=4 / 11, q_{2}=5 / 8, p_{1}=9 / 11, p_{2}=7 / 8, \lambda_{1}=1 / 13, \lambda_{2}=1 / 15, n=2, m=2, a=\sqrt{2} / 10$, $T=\sqrt{2}, \sigma_{1}=1 / \sqrt{5}, \sigma_{2}=1 / \sqrt{7}, \tau_{1}=\sqrt{2} / 2, \tau_{2}=\sqrt{2} / 3, \eta_{1}=\sqrt{2} / 9, \eta_{2}=\sqrt{2} / 5, \xi_{1}=\sqrt{2} / 4$, $\xi_{2}=\sqrt{2} / 7, \alpha_{1}=1 / 5, \alpha_{2}=-1 / 7, \beta_{1}=2 / 9, \beta_{2}=-1 / 6, \rho_{1}=\sqrt{3} / 2, \rho_{2}=7 / 11, \gamma_{1}=4 / 9, \gamma_{2}=1 / 9$, and $(1 / 2)+(|x| \sin (\pi t)) /\left((1+t)^{2}\right)+\left(|y| /\left(20(1+t)^{2}\right)\right)(|y| /(|y|+4)+1)$ and $(1 / 3)+(|x| /((4+$ $\left.\left.t)^{2}\right)\right)(|x| /(|x|+5)+1)+\left(\cos ^{2} y(t)\right) /\left(9(2+t)^{2}\right)$. We have

$$
\left|f\left(t, x_{1}, x_{2}\right)\right| \leq \frac{1}{2}+\frac{100}{(30+\sqrt{2})^{2}}\left|x_{1}\right|+\frac{25}{(10+\sqrt{2})^{2}}\left|x_{2}\right|
$$

and

$$
\left|g\left(t, x_{1}, x_{2}\right)\right| \leq \frac{1}{3}+\frac{120}{(40+\sqrt{2})^{2}}\left|x_{1}\right|+\frac{100}{9(20+\sqrt{2})^{2}}\left|x_{2}\right| .
$$

By using the Maple program, we can find that

$$
\Psi_{2}=\sum_{j=1}^{n} \frac{\beta_{j} \Gamma\left(q_{2}\right)\left(\log \frac{\xi_{j}}{a}\right)^{q_{2}+p_{2}+\gamma_{j}-1}}{\Gamma\left(q_{2}+p_{2}+\gamma_{j}\right)}-\frac{\sigma_{2} \Gamma\left(q_{2}\right)\left(\log \frac{\tau_{2}}{a}\right)^{q_{2}+p_{2}-1}}{\Gamma\left(q_{2}+p_{2}\right)} \simeq-0.5134156525 \neq 0 .
$$

Then the assumptions of Theorem 3.2 are satisfied with $u_{0}=1 / 2, u_{1}=100 /(30+\sqrt{2})^{2}, u_{2}=$ $25 /(10+\sqrt{2})^{2}, v_{0}=1 / 3, v_{1}=120 /(40+\sqrt{2})^{2}, v_{2}=100 /\left(9(20+\sqrt{2})^{2}\right), M_{9} \simeq 1.733533545$, $M_{10} \simeq 0.1574090650, M_{11} \simeq 5.002175405, M_{12} \simeq 0.3666346318$, and

$$
\begin{aligned}
& l_{1}=u_{1} M_{9}+v_{1} M_{11}+M_{10} \simeq 0.6830503591<1, \\
& l_{2}=u_{2} M_{9}+v_{2} M_{11}+M_{12} \simeq 0.8204817323<1
\end{aligned}
$$

and

$$
l_{0}=\max \left\{1-l_{1}, 1-l_{2}\right\}=\max \{0.1795182677,0.3169496409\}=0.1795182677 .
$$

Thus all the conditions of Theorem 4.2 hold true and consequently by the conclusion of Theorem 4.2, the problem (4.7) has at least one solution on $[\sqrt{2} / 10, \sqrt{2}]$.

Competing interests

The authors declare that they have no competing interests.

\section{Authors' contributions}

All authors contributed equally in this article. They read and approved the final manuscript.

\section{Author details}

'Nonlinear Dynamic Analysis Research Center, Department of Mathematics, Faculty of Applied Science, King Mongkut's University of Technology North Bangkok, Bangkok, 10800, Thailand. ${ }^{2}$ Centre of Excellence in Mathematics, CHE, Si Ayutthaya Rd., Bangkok, 10400, Thailand. ${ }^{3}$ Department of Mathematics, University of loannina, Ioannina, 451 10, Greece. ${ }^{4}$ Nonlinear Analysis and Applied Mathematics (NAAM) Research Group, Department of Mathematics, Faculty of Science, King Abdulaziz University, P.O. Box 80203, Jeddah, 21589, Saudi Arabia.

\section{Acknowledgements}

This research is partially supported by the Centre of Excellence in Mathematics, the Commission on Higher Education, Thailand. We thank the reviewers for their constructive comments, which led to the improvement of the original manuscript. 


\section{References}

1. Samko, SG, Kilbas, AA, Marichev, Ol: Fractional Integrals and Derivatives: Theory and Applications. Gordon \& Breach, Yverdon (1993)

2. Podlubny, I: Fractional Differential Equations. Academic Press, San Diego (1999)

3. Kilbas, AA, Srivastava, HM, Trujillo, JJ: Theory and Applications of Fractional Differential Equations. North-Holland Mathematics Studies, vol. 204. Elsevier, Amsterdam (2006)

4. Baleanu, D, Diethelm, K, Scalas, E, Trujillo, JJ: Fractional Calculus Models and Numerical Methods. Series on Complexity, Nonlinearity and Chaos. World Scientific, Boston (2012)

5. Ahmad, B, Nieto, JJ: Riemann-Liouville fractional integro-differential equations with fractional nonlocal integral boundary conditions. Bound. Value Probl. 2011, 36 (2011)

6. Ahmad, B, Ntouyas, SK, Alsaedi, A: New existence results for nonlinear fractional differential equations with three-point integral boundary conditions. Adv. Differ. Equ. 2011, Article ID 107384 (2011)

7. Ahmad, B, Ntouyas, SK, Alsaedi, A: A study of nonlinear fractional differential equations of arbitrary order with Riemann-Liouville type multistrip boundary conditions. Math. Probl. Eng. 2013, Article ID 320415 (2013)

8. Ahmad, B, Nieto, JJ: Boundary value problems for a class of sequential integrodifferential equations of fractional order. J. Funct. Spaces Appl. 2013, Article ID 149659 (2013)

9. Agarwal, RP, Zhou, Y, He, Y: Existence of fractional neutral functional differential equations. Comput. Math. Appl. 59, 1095-1100 (2010)

10. Baleanu, D, Mustafa, OG, Agarwal, RP: On $L^{P}$-solutions for a class of sequential fractional differential equations. Appl. Math. Comput. 218, 2074-2081 (2011)

11. O'Regan, D, Stanek, S: Fractional boundary value problems with singularities in space variables. Nonlinear Dyn. 71 , 641-652 (2013)

12. Zhang, L, Ahmad, B, Wang, G, Agarwal, RP: Nonlinear fractional integro-differential equations on unbounded domains in a Banach space. J. Comput. Appl. Math. 249, 51-56 (2013)

13. Liu, X, Jia, M, Ge, W: Multiple solutions of a $p$-Laplacian model involving a fractional derivative. Adv. Differ. Equ. 2013, $126(2013)$

14. Hadamard, J: Essai sur l'etude des fonctions donnees par leur developpment de Taylor. J. Math. Pures Appl. 8, 101-186 (1892)

15. Butzer, PL, Kilbas, AA, Trujillo, JJ: Compositions of Hadamard-type fractional integration operators and the semigroup property. J. Math. Anal. Appl. 269, 387-400 (2002)

16. Butzer, PL, Kilbas, AA, Trujillo, JJ: Fractional calculus in the Mellin setting and Hadamard-type fractional integrals. J. Math. Anal. Appl. 269, 1-27 (2002)

17. Butzer, PL, Kilbas, AA, Trujillo, JJ: Mellin transform analysis and integration by parts for Hadamard-type fractional integrals. J. Math. Anal. Appl. 270, 1-15 (2002)

18. Kilbas, AA: Hadamard-type fractional calculus. J. Korean Math. Soc. 38, 1191-1204 (2001)

19. Kilbas, AA, Trujillo, JJ: Hadamard-type integrals as G-transforms. Integral Transforms Spec. Funct. 14, $413-427$ (2003)

20. Glushak, AV, Manaenkova, TA: Direct and inverse problems for an abstract differential equation containing Hadamard fractional derivatives. Differ. Equ. 47, 1307-1317 (2011)

21. Kassim, MDA: Well-posedness for a Cauchy fractional differential problem with Hilfer type fractional derivative. PhD thesis, King Fahd University of Petroleum and Minerals, Saudi Arabia (2011)

22. Bazhlekova, E: Fractional evolution equations in Banach spaces. PhD thesis, Eindhoven University of Technology, Eindhoven (2001)

23. Kostic, M: Abstract Volterra Integro-Differential Equations. CRC Press, Boca Raton (2015)

24. Prüss, J: Evolutionary Integral Equations and Applications. Birkhäuser, Basel (1993)

25. Coffey, WT, Kalmykov, YP, Waldron, JT: The Langevin Equation, 2nd edn. World Scientific, Singapore (2004)

26. Lim, SC, Li, M, Teo, LP: Langevin equation with two fractional orders. Phys. Lett. A 372, 6309-6320 (2008)

27. Lim, SC, Teo, LP: The fractional oscillator process with two indices. J. Phys. A, Math. Theor. 42, Article ID 065208 (2009)

28. Uranagase, M, Munakata, T: Generalized Langevin equation revisited: mechanical random force and self-consistent structure. J. Phys. A, Math. Theor. 43, Article ID 455003 (2010)

29. Denisov, SI, Kantz, H, Hänggi, P: Langevin equation with super-heavy-tailed noise. J. Phys. A, Math. Theor. 43, Article ID $285004(2010)$

30. Lozinski, A, Owens, RG, Phillips, TN: The Langevin and Fokker-Planck equations in polymer rheology. In: Handbook of Numerical Analysis, vol. 16 pp. 211-303 (2011)

31. Lizana, L, Ambjörnsson, T, Taloni, A, Barkai, E, Lomholt, MA: Foundation of fractional Langevin equation: harmonization of a many-body problem. Phys. Rev. E 81, Article ID 051118 (2010)

32. Gambo, YY, Jarad, F, Baleanu, D, Abdeljawad, T: On Caputo modification of the Hadamard fractional derivative. Adv. Differ. Equ. 2014, 10 (2014)

33. Ahmad, B, Eloe, PW: A nonlocal boundary value problem for a nonlinear fractional differential equation with two indices. Commun. Appl. Nonlinear Anal. 17, 69-80 (2010)

34. Ahmad, B, Nieto, JJ, Alsaedi, A, El-Shahed, M: A study of nonlinear Langevin equation involving two fractional orders in different intervals. Nonlinear Anal., Real World Appl. 13, 599-606 (2012)

35. Tariboon, J, Ntouyas, SK, Thaiprayoon, C: Nonlinear Langevin equation of Hadamard-Caputo type fractional derivatives with nonlocal fractional integral conditions. Adv. Math. Phys. 2014, Article ID 372749 (2014)

36. Diethelm, K: The Analysis of Fractional Differential Equations. Springer, Berlin (2010)

37. Granas, A, Dugundji, J: Fixed Point Theory. Springer, New York (2003) 